DRAFT VERSiOn JANUARY 15, 2018

Preprint typeset using $\mathrm{L}^{A} \mathrm{~T}_{\mathrm{E}} \mathrm{X}$ style emulateapj v. 08/22/09

\title{
THE ASYMPTOTIC FORM OF COSMIC STRUCTURE: SMALL SCALE POWER AND ACCRETION HISTORY
}

\author{
Michael T. Busha, August E. Evrard ${ }^{1}$, Fred C. Adams ${ }^{1}$ \\ Michigan Center for Theoretical Physics \\ Physics Department, University of Michigan, Ann Arbor, MI 48109 \\ mbusha, evrard,fca@umich.edu \\ Draft version January 15, 2018
}

\begin{abstract}
We explore the effects of small scale structure on the formation and equilibrium of dark matter halos in a universe dominated by vacuum energy. We present the results of a suite of four N-body simulations, two with a $\Lambda$ CDM initial power spectrum and two with WDM-like spectra that suppress the early formation of small structures. All simulations are run into to far future when the universe is $64 h^{-1}$ Gyr old, long enough for halos to essentially reach dynamical equilibrium. We quantify the importance of hierarchical merging on the halo mass accretion history, the substructure population, and the equilibrium density profile. We modify the mass accretion history function of Wechsler et al. (2002) by introducing a parameter, $\gamma$, that controls the rate of mass accretion, $d \ln M / d \ln a \propto a^{-\gamma}$, and find that this form characterizes both hierarchical and monolithic formation. Subhalo decay rates are exponential in time with a much shorter time scale for WDM halos. At the end of the simulations, we find truncated Hernquist density profiles for halos in both the CDM and WDM cosmologies. There is a systematic shift to lower concentration for WDM halos, but both cosmologies lie on the same locus relating concentration and formation epoch. Because the form of the density profile remains unchanged, our results indicate that the equilibrium halo density profile is set independently of the halo formation process.
\end{abstract}

Subject headings: cosmology:theory — large-scale structure of universe — dark matter

\section{INTRODUCTION}

Our understanding of cosmology has consolidated significantly in recent years and we are now approaching percent-level estimates of the most important cosmological parameters (e.g., Spergel et al. 2006). Using these parameters, numerical simulations of large scale structure provide excellent descriptions of the distribution and properties of dark matter halos. The next open question is to understand why non-linear structure takes the form that it does - a form that has been predicted by simulations and confirmed, more or less, by observational data. In particular, we would like to know how and why dark matter halos attain a nearly universal form for their density profiles, as first described by Navarro, Frenk, \& White 1997 (hereafter NFW). One aspect of this issue is understanding the importance of the method of mass accretion: How much does the final structure depend on accreting mass as virialized clumps as opposed to a continuum of diffuse material and how effectively does violent relaxation (Lvnden-Bell 1967) erase the memory of this accretion process.

The basic process for the buildup of structure in our cold dark matter (CDM) dominated universe is the hierarchical merging of collapsed structures (see, e.g., Press \& Schechter 1974; Aarseth et al. 1979; Blumenthal et al. 1984; Davis et al. 1985). This process creates small halos early in the universe which merge with each other while accreting material from their surroundings, eventually creating the large cluster-sized structures of today through a "bottom up" process.

\footnotetext{
${ }^{1}$ Astronomy Department, University of Michigan, Ann Arbor,
} MI 48109
For a time it was thought that light neutrinos might dominate the mass density, forcing galaxy formation to occur through a "top down" process (e.g., Bond \& Szalay 1983). In such a hot dark matter model, perturbations on small scales are washed out by free streaming, preventing the formation of the early low-mass seeds of hierarchical structure formation. Dark matter halos still form, but the first objects are large cluster-mass halos (Zel'dovich 1970; Doroshkevich \& Zel'dovich 1975). While popular in the 1970's because simulations reproduced the outline of the cosmic web that surveys were just beginning to map out (Thompson \& Gregory 1978), hot dark matter models have been ruled out based on observations of the galaxy distribution (White, Frenk, \& Davis 1983). Tuning the free-streaming mass-scale leads to WDM possibilities which suppress density perturbations below some (typically dwarf galaxy sized) scale. The most immediate effect of this suppression is to reduce the number of small halos and subhalos existing in large halos. Several numerical studies of WDM cosmologies have been carried out (e.g., Evrard \& Crone 1992; Avila-Reese et al. 2001; Bode. Ostriker, \& Turok 2001; Colín, Avila-Reese, \& Valenzuela 2000; White \& Croft 2000; Knebe et al. 2002, 2003), mostly in an attempt to explain the apparent lack of substructure in our local group as compared to predictions from $\Lambda$ CDM simulations.

Although CDM and hierarchical merging has emerged as the standard paradigm for structure formation, and is the key ingredient in setting the distribution of dark matter halos, it is still uncertain how important this process of mass accretion is in setting the internal proper- 
ties of dark matter halos. While some studies indicate that the accretion of substructures plays a significant role in setting the inner slope of the radial halo density profile (Ma \& Bovlan-Kolchin 2004), simulations of monolithic collapse events in WDM scenarios produce halos with global properties unchanged relative to similar structures in CDM cosmologies (Evrard \& Crone 1992. Moore et al. 1999). By truncating the initial power spectrum in an otherwise CDM simulation at some scale $k_{c}$ (with corresponding mass scale $M_{c}=4 / 3 \pi^{4} k_{c}^{-3} \bar{\rho}$ ), one can mimic WDM cosmologies and test the importance of hierarchical growth for establishing halo structure. Compared to the CDM model, where mass is continually accreted in dense clumps, WDM cosmologies have an early period of monolithic collapse, where large halos form out of a smooth background and relax with many fewer disruptions due to merger events. If the halo is in a dense enough region, this initial collapse is followed by hierarchical accretion. Previous simulations of this process (Moore et al. 1999; Bode, Ostriker, \& Turok 2001; Avila-Reese et al. 2001) have shown that the resulting density profile is virtually unchanged for halos well above the truncation scale.

Halos in a WDM cosmology are effectively a re-scaled versions of the first halos expected in a CDM cosmology. Most physical CDM candidates (i.e., SUSY-LSP's) have some intrinsic velocity dispersion, washing out perturbations on very small scales (much smaller than we are able to simulate on cosmological scales), effectively truncating the power spectrum at some very large $k$ (see Diemand, Moore, \& Stadel 2005; Gao et al. 2005 for discussions of such simulations). In this manner, studying WDM cosmologies can provide clues to the earliest collapse of CDM structures.

The discovery of the accelerated expansion of the universe (Perlmutter et al. 1998), potentially caused by a non-zero cosmological constant (the $\Lambda \mathrm{CDM}$ model), provides us with a mechanism for studying the true equilibrium structure of cosmic halos. Previous work (Adams \& Laughlin 1997; Nagamine \& Loeb 2003; Busha et al. 2003, 2005) has shown that, in the relatively near cosmic future of a $\Lambda$ CDM universe, structure formation and halo growth will come to a rapid end. This happens around $a \sim 3$, when $\Omega_{\Lambda} \approx 1$. At this time, exponential deSitter expansion causes mergers and accretion to stop and pushes existing halos further and further away from each other. From $a \sim 3$ beyond, no matter is left for halos to accrete, and they relax toward their asymptotic equilibrium state in effective isolation. These late-time halos experience no disruption from mergers or other events that frustrate the equilibrium, in contrast to the situation at the present epoch.

To gain insight into the question of the origin of the internal structure of dark matter halos, we have performed a set of simulations of cosmic structure, using both a standard $\Lambda$ CDM and truncated WDM-like power spectra. Simulations are run into the far future, allowing halos to relax toward equilibrium configurations. Our numerical simulations are described in $\S 2$. In $\S 3$, we compare the differences in the halo distribution for the two cosmologies, concentrating on the mass function, evolution of the power spectrum, and the formation of WDM halos with mass well below the truncation scale. In $\S 4$ we compare the internal structure of the halos in the
TABLE 1

SiMULATION PARAMETERS:

\begin{tabular}{cccc}
\hline$L\left[h^{-1} \mathrm{Mpc}\right]$ & $M_{p}\left[h^{-1} \mathrm{M}_{\odot}\right]$ & $\epsilon_{p}\left[h^{-1} \mathrm{Mpc}\right]$ & $N_{p}$ \\
\hline 200 & $3.97 \times 10^{10}$ & 0.04 & $256^{3}$ \\
50 & $6.20 \times 10^{8}$ & 0.01 & $256^{3}$ \\
\hline
\end{tabular}

two cosmologies, including the mass accretion histories (MAHs), the distribution of substructure, and the halo density profiles. Results and their implications are summarized and discussed in $\S 5$.

\section{SIMULATIONS}

We simulate the formation of dark matter halos in $\Lambda$-dominated CDM and WDM-like cosmologies with a suite of dark matter N-body simulations using the publicly available TreePM code Gadget 2.0 (Springel 2005). We use two $\Lambda$ CDM and WDM simulations with different mass resolutions for a total of four large-scale cosmological simulations. The simulation pairs at each mass resolution were created with the same initial phases so that the the large-scale environment would be unchanged. All simulations model a patch of space in a flat universe with current matter density $\Omega_{\mathrm{m}}=0.3$, vacuum density $\Omega_{\Lambda}=0.7$, Hubble parameter $H_{0}=70 \mathrm{~km} \mathrm{~s}^{-1} \mathrm{Mpc}^{-1}$ and power spectrum normalization $\sigma_{8}=0.9$, values consistent with the first year release of WMAP measurements of the CMB power spectrum (Spergel et al. 2003). The lower resolution simulations model periodic cubes of side length $L=200 \mathrm{~h}^{-1} \mathrm{Mpc}$ containing $N_{p}=256^{3}$ particles of mass $3.97 \times 10^{10} h^{-1} \mathrm{M}_{\odot}$ and gravitational softening length $\epsilon_{p}=40 h^{-1} \mathrm{kpc}$. The higher resolution simulations use cubes of side length $L=50 h^{-1} \mathrm{Mpc}$, containing $256^{3}$ particles with mass $6.20 \times 10^{8} h^{-1} \mathrm{M}_{\odot}$ and softening length $\epsilon_{p}=10 h^{-1} \mathrm{kpc}$. The softening scales quoted here correspond to their values at the present epoch and are held constant in comoving space for $a<1$, but become physical lengths for $a>1$ to prevent structures from being over-softened due to the exponential increase in $a$ during the deSitter phase. Table 1 lists these simulation parameters. All simulations were started at redshift $z=19$ (scale factor $a=0.05$ ) and were run into the far future, $a=100$. Although the starting redshift is somewhat late, it is consistent with the analysis by Power et al. (2003) for a simulation of our resolution, and should present no problem for the late-time results we are primarily concerned with. We store a total of 300 outputs equally spaced in $\log (a)$ for each simulation. At $a=100$ the universe is about $64 h^{-1} \mathrm{Gyr}$ old and structure formation has preceded to completion in a $\Lambda$ CDM cosmology (Busha et al. 2003; Nagamine \& Loeb 2003). The simulations were run on 16 nodes of a dual-Opteron Beowulf cluster at the University of Michigan.

The initial power spectrum for our CDM simulations was set using CMBFAST (Seljak \& Zaldarriaga 1996) in accordance with WMAP year 1 data (Spergel et al. 2003). Our second, WDM-like, simulation used a truncated version of this initial power spectrum,

$$
P_{t}(k)=C P_{0}(k) e^{-\left(k / k_{c}\right)^{2}},
$$

where $k_{c}$ is the truncation scale and $C$ a normalization 


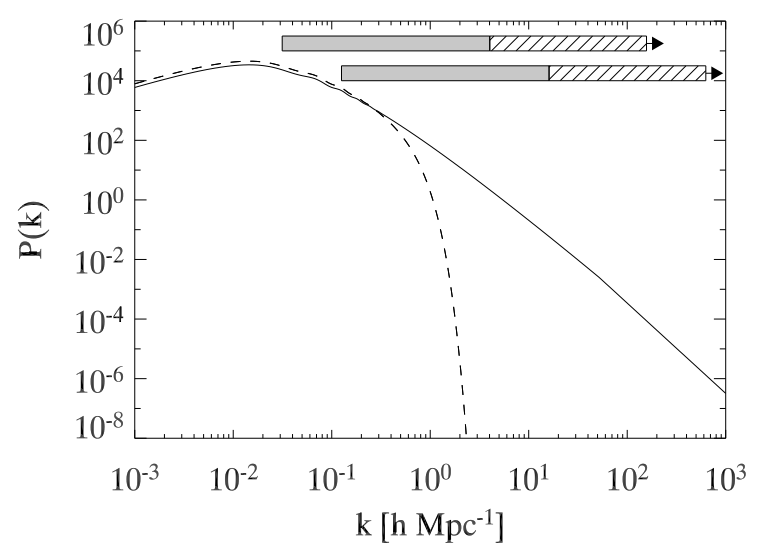

FIG. 1. - The input power spectra for the $\Lambda$ CDM (sold line) and WDM (dashed line) simulations. The $\Lambda$ CDM spectrum is calculated using CMBFAST . The WDM model adds an exponential cutoff to the $\Lambda \mathrm{CDM}$ model at a mass scale of $8.09 \times 10^{13} h^{-1} \mathrm{M}_{\odot}$. Both spectra were normalized so that $\sigma_{8}=0.9$. The light gray boxes represent the range between the fundamental and Nyquist frequencies for the small (top) and large (bottom) volume runs. The hatched-line boxes show the range between the Nyquist and softening frequencies for $a \leq 1$. For $a>1$, the softening is constant in the physical frame and its corresponding wavenumber grows with $k \propto a$ in this comoving representation.

coefficient that allows us to set $\sigma_{8}$. Figure 1 plots our input spectra, with $P_{0}$ as the solid line and $P_{t}$ as the dashed line. For our simulations, we choose a truncation scale of $k_{c}=0.511 \mathrm{~h} \mathrm{Mpc}^{-1}$, which corresponds to a mass scale of $M_{c}=8.09 \times 10^{13} h^{-1} \mathrm{M}_{\odot}$ (2037 particles for our lower resolution simulations and 130,396 at the higher resolution). This truncated spectrum was re-normalized to $\sigma_{8}=0.9$ so that high mass halo abundances would be similar. As noted earlier, phase information was retained for each resolution pair, resulting in similar large scale structures (Figure 21) and allowing us to identify corresponding halos in the two cosmologies.

Equation (1) represents a transfer function that differs from the standard transfer function for a WDM cosmology. Generally, the mass of the WDM particle, $m_{W}$ sets a free streaming length, $\quad R_{f}=0.2\left(\Omega_{W} h^{2}\right)^{1 / 3}\left(m_{W} / 1 \mathrm{keV}\right)^{-4 / 3} \mathrm{Mpc}$ (Sommer-Larsen \& Dolgov 2001), which approximates the WDM power spectrum through the relation (Bardeen et al. 1986)

$$
P_{W D M}(k)=\exp \left[-k R_{f}-\left(k R_{f}\right)^{2}\right] P_{C D M}(k) .
$$

This spectrum has a slightly more gradual cutoff than equation (11). It should be noted that we refer to our truncated models as "WDM" cosmologies even through they were not created using this transfer function. For reference, our truncated model most closely approximates a WDM cosmology with $m_{W D M}=0.13 \mathrm{keV}$ and $R_{f}=1.6 \mathrm{Mpc}$.

The evolution of the resulting density fields of the simulations are shown in Figures 2 and 3 Figure 2 shows a slice the comoving density field from our smaller volume CDM and WDM simulations at $a=0.3,1,3$, and 100. The differences between these two models are striking, especially at $a=0.3$, where the CDM cosmology exhibits a well-formed web with an abundance of small halos. The WDM cosmology, in contrast, has a mostly uniform density, with only one visible halo and a handful of weak filamentary structures making up the cosmic web. A clear cosmic web does rapidly develop in the WDM simulation, however, and by $a=1$ similar large scale structures are present in both cosmologies, even though there is a strong suppression of small halos in the WDM filaments. By $a=3$, the large scale density field is set and undergoes little evolution from $a=3$ to 100. Once the cosmological constant becomes dominant the growth function saturates, ending halo formation and freezing the comoving web. Halos continue to contract in this comoving picture, and by $a=100$ they consist of small, tightly bound knots along and at the intersection of filaments.

Figure 3 shows the evolution of the density field in a fixed physical region. Here, we focus on the evolution of a particular CDM halo with mass $M_{200}=$ $5.38 \times 10^{14} h^{-1} \mathrm{M}_{\odot}$ and its counterpart in the WDM cosmology (see $\S 3.3$ ). While much of the late time growth is identical (such as the major merger around $a=1$ ), the initial formation processes differ substantially. At $a=0.3$, there are many low mass CDM progenitor halos present, while the WDM halo looks like a weak (uncollapsed) perturbation in an otherwise smooth background. This difference is manifest throughout all plotted epochs by the persistent lack of substructure in the WDM halo at the three later epochs.

One way to quantify the expected suppression of hierarchical buildup is to look at the critical mass scale, $M_{*}(a)$, where one expects a perturbation to go non-linear and collapse, defined through the relation

$$
\sigma\left[M_{*}(a)\right]=\frac{\delta_{c}}{D(a)} .
$$

Here, $\sigma(M)=(2 \pi)^{-3} \int_{0}^{\infty} P(k) \widetilde{W}^{2}(M, k) d^{3} k, \widetilde{W}$ is the Fourier transformation of the top-hat window function, $D(a)$ is the linear growth function, and $\delta_{c}=1.686$ is the linearly extrapolated criterion for collapse of an overdense perturbation (Press \& Schechter 1974; Peebles 1980). The factor $D(a)$ can be calculated numerically from the expression

$$
D(a) \propto H(a) \int_{0}^{a} \frac{d a^{\prime}}{\left[a^{\prime} H\left(a^{\prime}\right)\right]^{3}},
$$

and is normalized such that $D(1)=1$. The left panel of Figure 4 shows the amplitude of $2 \sigma(M)$ perturbations as a function of $M$ at the present epoch. The horizontal dotted line shows the critical scale, $\delta_{c}=1.686$. The right panel of Figure 4 shows the evolution of $M_{2 \sigma}(a)$ - the mass of a $2 \sigma$ perturbation that goes nonlinear as a function of cosmic time. In both cases, the solid line represents the full power spectrum model and the dashed line represents the truncated power spectrum model. We consider $2 \sigma$ perturbations because, in our WDM cosmology, $1 \sigma$ perturbations do not collapse until $a=1.33$ and are prevented from growing substantially by the cosmological constant. The normalization $\sigma_{8}=0.9$ boosts the power at large mass $\left(M>10^{14} h^{-1} \mathrm{M}_{\odot}\right)$ in the WDM model relative to the CDM case and causes the largest perturbations to collapse slightly earlier and with larger asymptotic masses in the WDM model. We also expect no structures in the WDM cosmology for $a<0.4$, agree- 


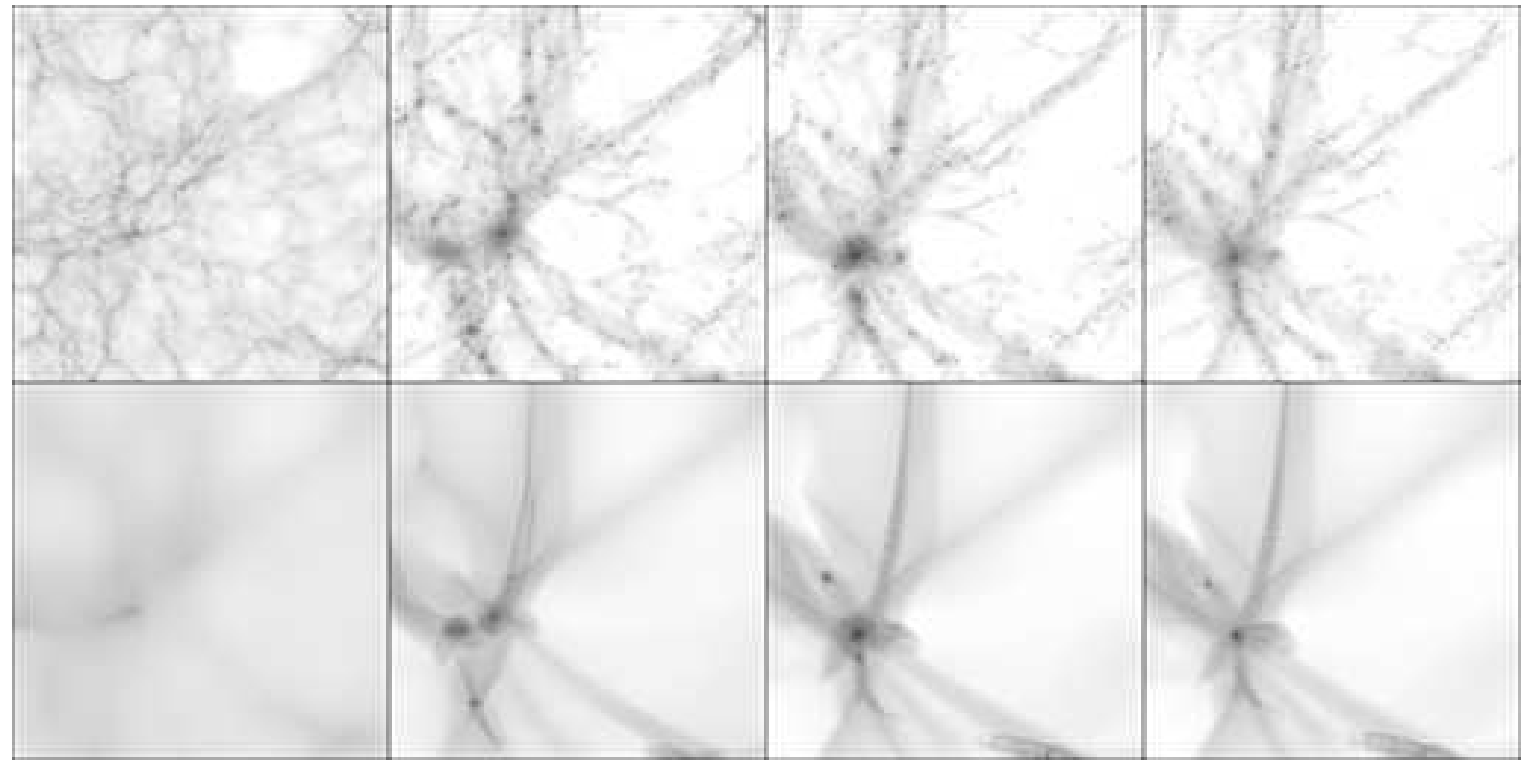

FIG. 2. - The density fields of comoving slices of the cosmic web at $a=0.3,1,3$, and 100 (columns, left to right) of a $\Lambda$ CDM (top) and WDM (bottom) cosmologies from our small volume simulations. The side length for each image is $35 h^{-1} \mathrm{Mpc}$, with thickness $5 h^{-1} \mathrm{Mpc}$. The grey-scale is proportional to $\log (\rho / \bar{\rho})$.

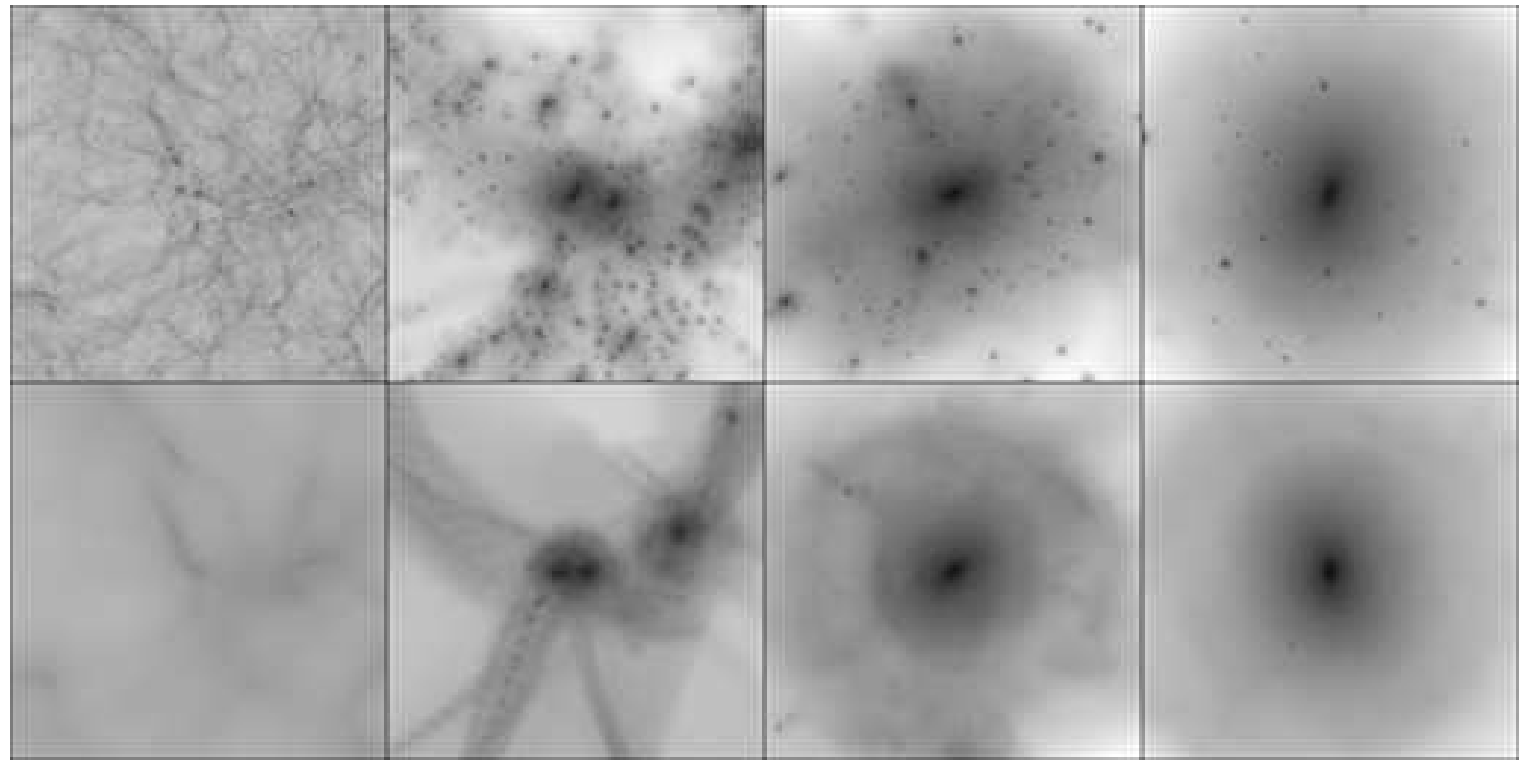

FIG. 3. - The density field around a large halo at $a=0.3,1,3$, and 100 (columns, left to right) from our small volume simulations. The top row shows the largest halo from the $\Lambda$ CDM cosmology $\left(M_{200}=5.38 \times 10^{14} h^{-1} \mathrm{M}_{\odot}\right.$ at $\left.a=100\right)$ and the bottom row shows the corresponding WDM halo $\left(M_{200}=5.24 \times 10^{14} h^{-1} \mathrm{M}_{\odot}\right.$ at $\left.a=100\right)$. The side length for each image is $12 h^{-1} \mathrm{Mpc}$, with thickness $6 h^{-1} \mathrm{Mpc}$ in physical units. The grey-scale is proportional to $\log \left(\rho / \rho_{c}\right)$.

ing with Figure 3 in which the most massive halo of the simulation has yet to form a coherent structure prior to this epoch.

Dark matter halos in our simulations are identified using a standard friends-of-friends (FOF) algorithm with linking length 0.15 times the inter-particle spacing. Halo centers are identified as the most bound particle of the resulting group. As a mass measure capable of spanning both early and late times we use $M_{200}$, the mass of all particles inside a sphere of radius $r_{200}$ with over-density 200 times the critical density, $\rho_{c}$. In previous work, we found the $M_{200}$ provides a good proxy for the asymptotic halo mass and is roughly half the value of the ultimate halo mass, $M_{200} \simeq 0.5 M_{\text {halo }}$ (Busha et al. 2005). The halo velocity is defined to be the center of mass velocity of all particles within $r_{200}$.

Subhalos were identified using the SUBFInd routine (Springel et al. 2001). This routine works on top of an FOF group and identifies density maxima within halos from an SPH smoothing kernel that uses the distance to the $32^{\text {nd }}$-nearest neighbor to obtain local density estimates. Subhalos are then selected as locally overdense regions containing at least 20 bound particles. The largest subhalo identified by SUBFIND is actually the host halo of the FOF group. At $a=100$, this host halo should correspond to the actual equilibrated halo as defined in Busha et al. (2005), minus any locally bound subhalos. A comparison between these mass estimates shows good 

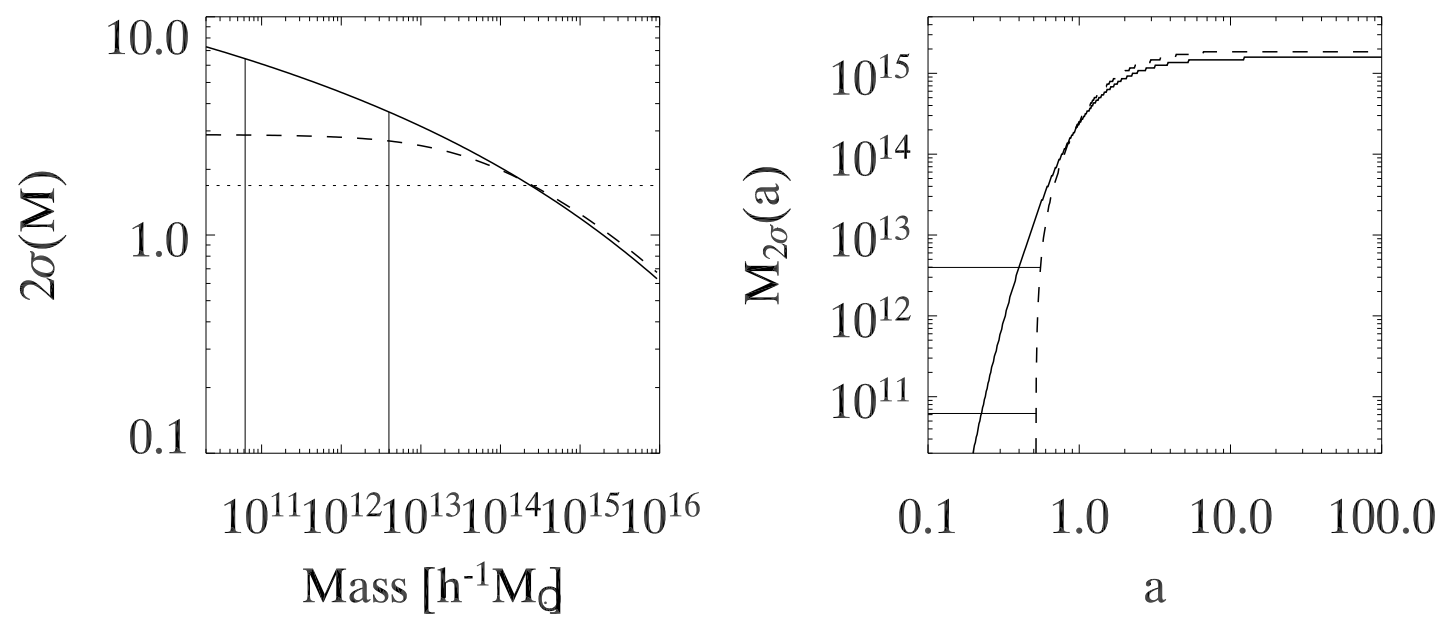

FIG. 4. - Left: Rare $(2 \sigma)$ perturbation amplitude as a function of mass at $a=1$ for the $\Lambda$ CDM (solid line) and WDM (dashed line) models. The vertical lines show the mass scales for 100 particles at the two resolutions. Right: Characteristic collapsed mass as a function of scale factor for the $\Lambda$ CDM (solid line) and WDM (dashed line) cosmologies. The solid horizontal lines show the mass scales for 100 particles at the two resolutions.

agreement, with the masses agreeing to within $1 \%$.

Throughout this paper, we use several scale radii. Halo sizes are defined using $r_{200}$ (see above) and $r_{\text {halo }}$, the spherical radius containing all bound particles (which is only defined for $a \gtrsim 5$, see Busha et al. 2005). Additionally, we fit NFW and Hernquist density profiles to our halos,

$$
\begin{aligned}
\rho_{N F W} & =\frac{4 \rho_{s}}{r / r_{s}\left(1+r / r_{s}\right)^{2}}, \\
\rho_{H e r n} & =\frac{\rho_{0}}{r / r_{c}\left(1+r / r_{c}\right)^{3}},
\end{aligned}
$$

which adds the scales $r_{s}$ (the radius where the best fit NFW profile has logarithmic slope -2 ) and $r_{c}$ (where the best fit Hernquist profile has slope -2.5). Generally, we find that $r_{\text {halo }}=4.6 r_{200}$ and $r_{s}=0.4 r_{c}$ ( Busha et al. 2005).

\section{COMPARISONS OF THE DARK MATTER DISTRIBUTION}

In this section we compare properties of the distribution of dark matter halos in our simulations, including the evolution of the power spectrum, the halo mass function, the correspondence between CDM and WDM halos, and the formation of WDM halos below the truncation scale.

\subsection{Evolution of the Power Spectrum}

Figure 5 shows the evolution of the power spectrum for all four of our simulations at the epochs $a=0.3,1,3$, and 100. The solid lines represent the CDM cosmologies and the dashed lines the WDM spectrum. The power spectrum is shown in dimensionless units, $\Delta^{2}(k) \propto k^{3} P(k)$. Power spectra for the large and small volume simulations are combined, allowing us to probe a larger range in $k$. The arrows represent the softening lengths for the large and small volume simulations at the plotted epoch. The collapse of non-linear structure creates substantial power beyond the Nyquist frequency, which we measure using the tiling method of Jenkins et al. (1998). The spectra are plotted from the fundamental simulation frequency out to a wavenumber where the shot noise of a Poisson distribution of particles becomes comparable to the measured power.

At $a=0.3$, when non-linear structure formation is in its early stages, the power spectrum of the WDM model is heavily truncated above $k_{c}$. By the present epoch, much of this suppression has disappeared due to power transfer from collapsing structures, and the WDM cosmology matches the CDM model almost perfectly at low and intermediate wavenumber, up to an order of magnitude above $k_{c}$. Relatively little happens to the power spectrum beyond $a=1$. As noted earlier, the dominance of $\Lambda$ halts the growth of structure beyond $a \sim 3$ and causes the power spectrum to freeze after only a modest amount of additional evolution. Since Figure 5 plots the power spectrum in comoving space, beyond $a=1$ the expansion of the universe transfers power to larger scales with no real change in the shape of the spectrum. For most of the measurable range, $0.1<k<100 \mathrm{hMpc}^{-1}$, the power spectrum is well characterized by the expected power law, $\Delta^{2} \propto k^{3}$, caused by the freezing of structure in an expanding universe. Collapse, however, has not managed to elevate the WDM power spectrum up to that of the CDM model at all scales by $a=3$, and a small suppression of power at the highest wavenumbers persists at $a=100$. This suppression is due to the lack of low mass halos. If we measure the halo power the spectrum using only halos with $M_{200}>M_{c}$ we recover spectra that are identical at the few percent level, which indicates that the distribution of halos on scales greater than $M_{c}$ is statistically unchanged.

The evolution of the power spectrum in WDM cosmologies was studied previously by White \& Croft (2000) and Knebe et al. (2002). These studies present results similar to ours. By $a \sim 0.5$, non-linear collapse has boosted the truncated portion of the power spec- 


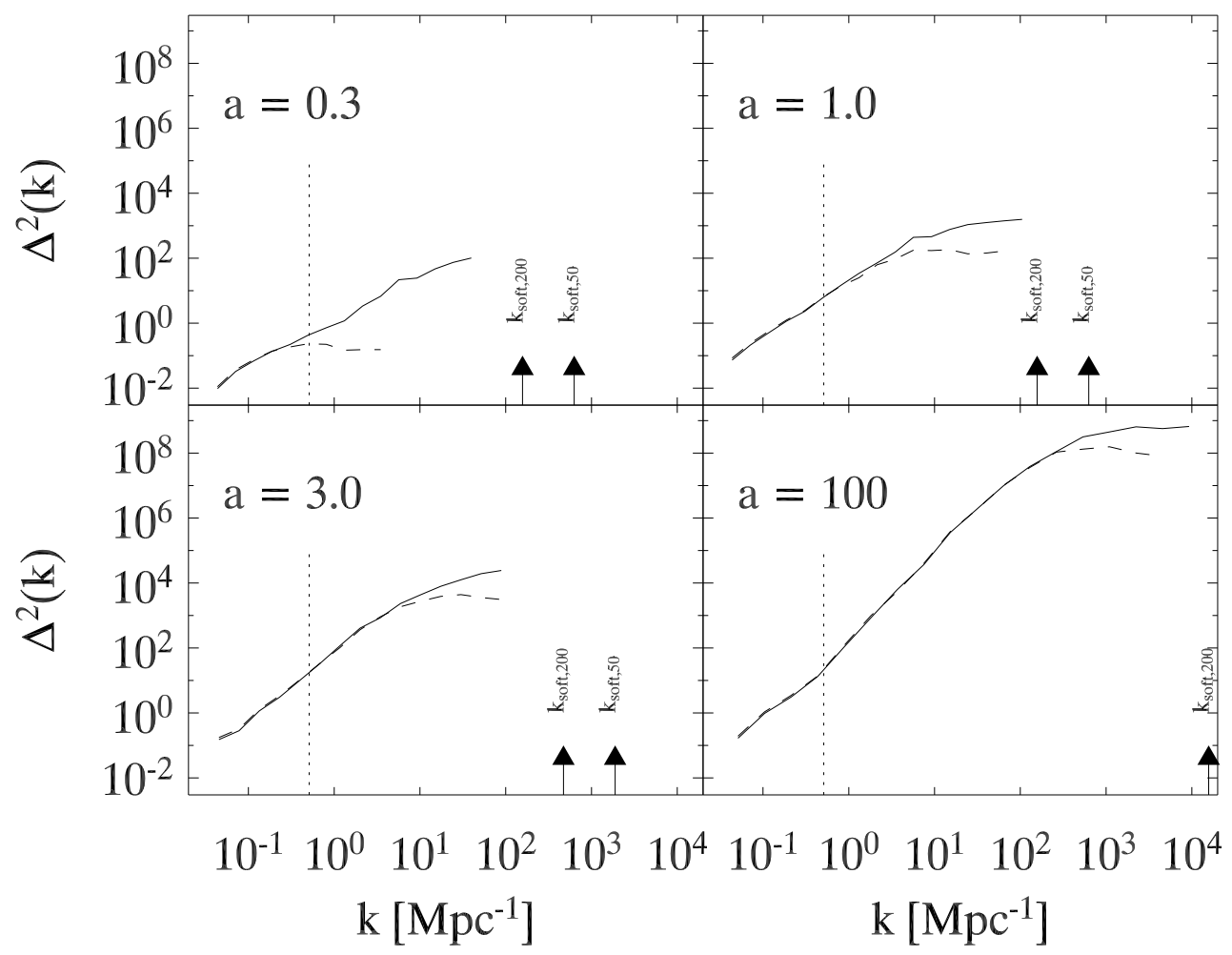

FIG. 5.- The evolution of the power spectrum for halos in CDM (solid lines) and WDM (dashed lines) cosmologies. The spectra are shown at $a=0.3,1,3$, and 100. Power spectra from the small and large volume realizations are laid on top of each other. The dotted vertical lines represent the truncation scale, $k_{c}$, and the arrows show the softening scales for the large and small volume runs. The spectra are plotted from the fundamental frequency of the simulation volume to the frequency where the shot noise becomes comparable to the measured power, generally $\sim 0.1 k_{\text {soft }}$.

trum substantially. agreeing with a $\Lambda$ CDM model for $k<10 h \mathrm{Mpc}^{-1}$ with only a slight suppression for larger $k$.

\subsection{Mass Function}

Figure 6 shows the mass function for our large volume CDM and WDM simulations at $a=1$ and 100. Errors are calculated assuming Poisson statistics and the vertical dotted line is the truncation scale, $M_{c}$. Here, the dark lines represent the $\Lambda \mathrm{CDM}$ model and the light lines the WDM version. For comparison, the figure also shows the Jenkins Mass Functions (JMF, Jenkins et al. (2001)) as dashed lines. The JMF is defined via

$$
\begin{aligned}
\frac{d n_{J M F}(M, a)}{d \ln (M)}= & A \frac{\bar{\rho}}{M} \frac{\mathrm{d} \ln \sigma^{-1}(M, a)}{d \ln M} \times \\
& \exp \left[-\left|\ln \sigma^{-1}(M, a)+B\right|^{\epsilon}\right],
\end{aligned}
$$

where $A, B$, and $\epsilon$ are fitting parameters. Two mass values are used in this plot to compare with published JMF parameters: FOF masses with a linking length $b=$ 0.164 (upper curves, Jenkins et al. 2001) and $M_{200}$ from a spherical overdensity groupfinder (SO, lower curves, Evrard et al. 2002). The fitting parameters are listed in Table 2. For the $a=100$ spherical overdensity JMF, we used the $\Omega_{M}=0$ parameters from Evrard et al. (2002). Although not shown, The Sheth \& Tormen mass function with published parameters (Sheth \& Tormen
1999) agrees quite well with the JMF FOF mass function at all epochs. While the agreement between FOF masses in the CDM cosmology and equation (7) is good for $a=1$, a substantial mass excess is present in the mass range $10^{13}<M_{\mathrm{FOF}}<5 \times 10^{14} h^{-1} \mathrm{M}_{\odot}$ at $a=100$. Additional simulations of this cosmology confirm that this excess is significant and not a result of one particular realization. The use for FOF masses in measuring the mass function does create difficulties in the far future because the FOF groupfinder identifies isosurfaces relative to the background matter density, which is dropping rapidly from the exponential expansion. Compared with the critical density, the physical density isosurface identified by a FOF groupfinder is $\rho_{\mathrm{FOF}}=\left[\left(a^{6} \Omega_{m, 0}\right) /\left(\Omega_{m, 0}+a^{3} \Omega_{\Lambda, 0}\right)\right] b^{-3} \rho_{\text {crit }}$. By $a=3$, $\rho_{\mathrm{FOF}}=3.5 \rho_{\text {crit }}$, which includes a substantial amount of material outside the virialized region of a halo that is unbound and being pulled away by the Hubble flow (Busha et al. 2005). At late epochs, $M_{\mathrm{FOF}}$ is a poor proxy for the actual (virialized) mass of a halo. The $M_{200}$ mass function, however, does not suffer from this defect. The $a=1$ result agrees with Evrard et al. (2002) to $\sim 20 \%$ in number, approximately the quoted statistical accuracy. The parameters are slightly off, however, due to insufficient mass resolution of the simulation. The $a=100$ mass function does provide a substantially better agreement than the FOF mass function, fitting to within about $2 \sigma$ at all masses. 


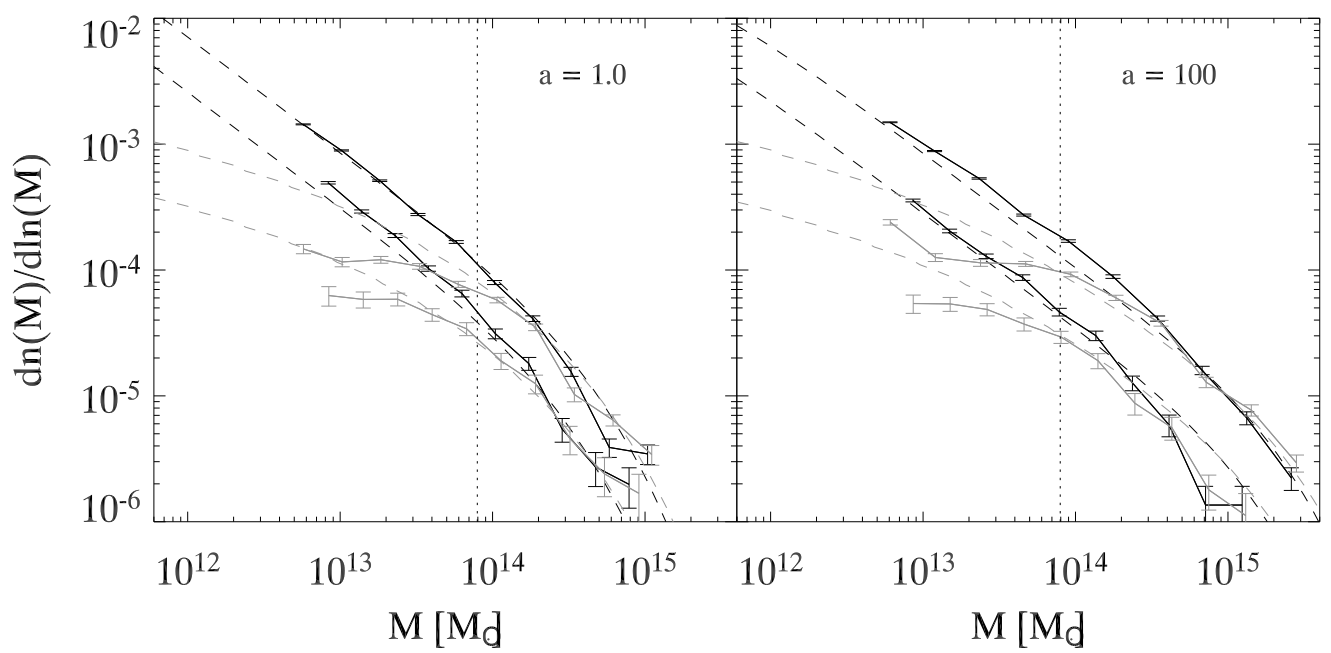

FIG. 6. - The mass function for the CDM and WDM cosmologies (dark and light curves) at $a=1$ and 100 for our larger volume simulations. The upper curves show the $\mathrm{FOF}(0.164)$ mass function and the lower curves the $\mathrm{SO}(200)$ mass function. The $\mathrm{FOF}(0.164)$ function has been offset vertically for clarity. The vertical dotted line shows the truncation scale. Error bars assume Poisson statistics.

TABLE 2

Mass Function Parameters:

\begin{tabular}{cccc}
\hline Mass Function & $A$ & $B$ & $\epsilon$ \\
\hline JMF $-\mathrm{FOF}(0.164)$ & 0.301 & 0.64 & 3.88 \\
$\mathrm{JMF}-\mathrm{SO}(200, a=1)$ & 0.220 & 0.73 & 3.86 \\
$\mathrm{JMF}-\mathrm{SO}(200, a=100)$ & 0.199 & 0.76 & 3.90 \\
\hline
\end{tabular}

The mass function for the WDM cosmology exhibits a striking suppression for low masses, beginning slightly above the the truncation scale, $M_{c}$ (dotted vertical line). An unexpected upturn appears in the WDM model at the lowest masses ( $\lesssim 50$ particles) for FOF halos. Bode. Ostriker. \& Turok (2001) and Knebe et al. (2002) claim that this behavior is a result of physical halos forming through fragmentation from Jeans instability. However, such an upturn is not present in the SO mass function and we show evidence in the appendix that it is actually a numerical artifact of the FOF groupfinder. We have also calculated JMF and ST fits for our WDM cosmology, which do not fit as well as in the $\Lambda$ CDM cosmology. Both the JMF and ST mass functions strongly over-predict the abundance of halos with masses $M<M_{c}$. The poor fit in the range $10^{13} h^{-1} \mathrm{M}_{\odot}<M<8 \times 10^{13} h^{-1} \mathrm{M}_{\odot}$ should not be surprising because equation (7) was motivated by a perturbation collapse threshold, similar to the Press-Schechter (1974) model, which uses spherical collapse to determine a collapse epoch. As we discuss in the next section, WDM halos with mass below $M_{c}$ form out of larger mass perturbations that do not follow this model, at odds with the assumptions of Press-Schechter.

\subsection{Halo Correspondence}

Because we used the same phases in constructing the initial conditions, we can cross-match halos in the CDM and WDM cosmologies using a Lagrangian scheme. We select a FOF halo in one cosmology (usually $\Lambda \mathrm{CDM}$ ) and identify for the largest halo in the other cosmology containing at least $50 \%$ of particles of the selected halo. When starting with a $\Lambda \mathrm{CDM}$ halo, we don't allow any two WDM halos to be identified with the same $\Lambda \mathrm{CDM}$ halo. This simple method is robust for massive systems, and corresponding halos are found for $98 \%$ of all halos with $M_{200}>M_{c}=8.08 \times 10^{13} h^{-1} \mathrm{M}_{\odot}$. The "missing" halos are lost because the smoothing of the power spectrum causes distinct halos in the CDM cosmology to form as single halos in the WDM run. Our requirement that each WDM halo have only a single CDM counterpart prevents all but the most massive of these CDM halos from having a WDM counterpart. If we relax this correspondence requirement, all CDM halos with $M_{F O F}>M_{c}$ have WDM counterparts. Figure 7 shows the masses of corresponding halos in the WDM and $\Lambda \mathrm{CDM}$ models. At higher masses $\left(M_{200}>2 M_{c}\right)$ the masses are similar, $M_{200, C D M} \approx M_{200, W D M}$ with about a $6 \%$ scatter. As the mass falls below the truncation scale, $M_{c}$, the WDM halos become less and less massive relative to their CDM counterparts, eventually disappearing altogether. There are a few extreme outliers from the general relationship. Halos with a low $M_{W D M} / M_{C D M}$ (the three halos with $M_{200, C D M} \sim 10^{14} h^{-1} \mathrm{M}_{\odot}$ and $\left.M_{200, W D M} \sim 2 \times 10^{12} h^{-1} \mathrm{M}_{\odot}\right)$ are CDM halos in filaments that never completely collapse in the WDM cosmology. Most of their particles are located in a spray along the filament, but there is a small WDM halo in the filament with $\sim 90 \%$ of its members in the CDM halo. The halo with the $M_{200, W D M} \gg M_{200, C D M}$ is a rare occurrence where many small $\mathrm{CDM}$ halos $(\sim 10)$ had merged into a single, much more massive halo in the WDM cosmology.

Also plotted in Figure 7 is the completeness function for identifying corresponding halos. Virtually all $\Lambda \mathrm{CDM}$ halos with $M_{200}>M_{c}$ have corresponding WDM halos, but the completeness function drops very rapidly for $M_{200}<M_{c}$, the mass range where halo formation is strongly suppressed. 


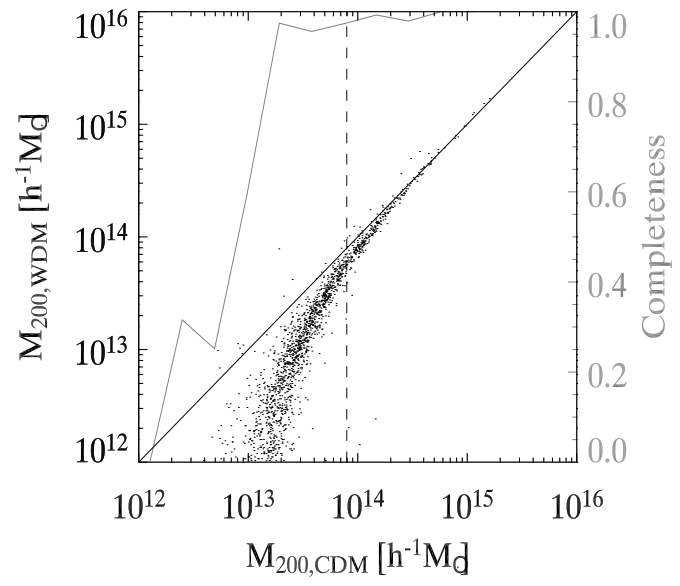

FIG. 7.- A comparison of the $M_{200}$ values for corresponding halos in the $\Lambda$ CDM and WDM runs at $a=100$. The dashed vertical line denotes the truncation scale. Halos with $M_{200} \gg M_{c}$ have roughly the same mass with a scatter of about $6 \%$. The light line shows the probability for finding a WDM halo corresponding to a $\Lambda$ CDM halo of a given mass.

\subsection{Sub-Truncation Scale Halos In WDM}

One surprising observation from the WDM simulations is that many halos form with mass scale $M_{200}$ well below the truncation scale, $M_{c}$. Although physical halos with $M_{200}<M_{c}=8.08 \times 10^{13} h^{-1} \mathrm{M}_{\odot}$ account for only a few percent of all collapsed mass, these halos represent a substantial population by number. Knebe et al. (2003) have shown that these halos appear in well-defined filaments and argue that they are the result of Jeans instability (Binney \& Tremaine 1987). Our results confirm that these halos form in filamentary structures, and we find that many of these halos have CDM counterparts, indicating that these low mass WDM halos are not numerical artifacts.

In order to understand how these sub-truncation scale halos form, we identify particles in small halos $\left(M_{200}<\right.$ $M_{c}$ ) in our small volume simulations at $a=100$ and trace them back to the initial conditions. We then locate the density peak nearest the center of mass of this distribution and measure the mass of the perturbation by finding the radius where spherical overdensity drops below $\delta_{c} D(a)$. For all halos in the WDM simulation, the perturbation mass is in the range $(0.2-2) \times 10^{14} h^{-1} \mathrm{M}_{\odot}=$ $0.25-2.5 M_{c}$. In particular, no halos form out of perturbations substantially smaller than our truncation scale, but sub-truncation scale halos do form out of large perturbations that do not fully collapse. The CDM simulation, by comparison, has small halos forming out of perturbations with masses anywhere between the final halo mass, $M_{\text {halo }}$, and $2 \times 10^{14} h^{-1} \mathrm{M}_{\odot}$, with the bulk of the halos $(80 \%)$ forming from perturbations with mass less than 4 times $M_{\text {halo }}(100)$. The distribution of perturbations with initial masses greater than $M_{c}$, however, is almost identical to that for the WDM cosmology. Low mass WDM halos are simply large perturbations that do not collapse completely, and behave the same as in a CDM cosmology. Such a trend was noted by Katz, Quinn, \& Gelb (1993), and our WDM halos seem to be an extreme case of the tendency for halos to form objects with a substantially different mass than their initial spherical collapse prediction.

\section{COMPARISON OF HALO PROPERTIES}

This section compares properties of individual halos in our CDM and WDM cosmologies. In particular, we focus on the mass accretion histories, subhalo abundance, and density profiles. In spite of some substantial and fundamental differences in the first two properties, the form of the radial density profiles is unchanged between the CDM and WDM cosmologies. Furthermore, halo concentrations follow the same relation with formation epoch in both models.

\subsection{Mass Accretion Histories}

One property for which we expect a clear difference between CDM and WDM halos is the halo mass accretion history (MAH). For the WDM model, the reduced merger activity should result in a smoother MAH since mass is primarily accreted in the form of diffuse material. The suppression of power at large $k$ also alters the characteristic collapse mass at low mass/early times (Figure 4, right panel). The implication is that halos will form later and more rapidly (in the sense of a larger $d \ln M / d \ln a$ ) in a WDM cosmology.

The MAHs are measured using a halo's most massive progenitor, where a progenitor is any halo at a preceding output in which at least $50 \%$ of the FOF particles end up in the subsequent halo. When comparing halos between the two runs, we first select CDM halos from a given mass range and then select either their WDM counterparts (as in Figure 7 ignoring CDM halos that have no match) or WDM halos from the same mass ranges. These selection methods are nearly degenerate for $M_{200}>M_{c}$, as shown in Figure 7. At lower CDM halo masses $M_{200, \mathrm{WDM}}$ is strongly suppressed, so the selection methods differ substantially. Generally, we prefer to consider halos of similar final masses so that we do not have to worry about halos with no counterpart.

Figure 8 shows MAHs for three halos in the full run (solid curves) and their matched halos in the truncated run (dashed curves). These individual halos were selected from our smaller volume run to have $M_{200} \gg$ $M_{c}$ (dark curves), $M_{200} \sim M_{c}$ (medium curves), and $M_{200}<M_{c}$ (light curves) at $a=100$ in the CDM cosmology. As expected, the halos in the truncated model have smoother MAHs during the initial halo growth phase and form slightly later, with these effects becoming more pronounced for smaller halos. The overall shape of the MAHs for the halos with $M_{200} \gg M_{c}$ is remarkably similar. While the most massive halo in the truncated model has no progenitor before $a \sim 0.2$, it grows quickly and catches up with the CDM halo by $a=0.7$. Afterwards, the two halos evolve almost identically, even undergoing the same major mergers around $a=1$ and 2 . These mergers happen slightly earlier in the WDM cosmology due to the increase in power at this scale from our normalization $\sigma_{8}=0.9$, which effectively starts the "cosmic clock" for these large halos at a later time. In contrast with the these late-time similarities, the MAHs at $a<0.7$ are substantially different. In the WDM scenario, a large mass perturbation will collapse more or less as a unit, as soon as it goes non-linear. This collapse creates a phase of smooth, rapid mass growth 


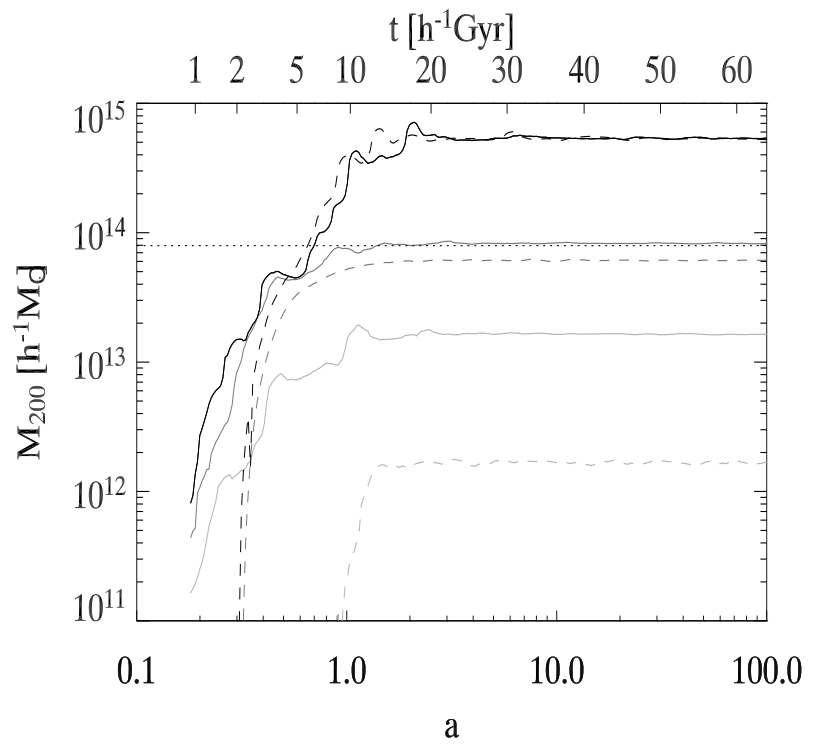

FIG. 8. - The accretion history for three individual halos in the full run (solid curves) and their corresponding halos in the truncated run (dotted curves). The halos were selected with $M_{200} \gg M_{c}$ (dark curves), $M_{200} \sim M_{c}$ (medium curves), and $M_{200} \ll M_{c}$ (light curves) in the run with the full power spectrum. The dotted horizontal line represents $M_{c}$.

with $d \ln M_{\mathrm{WDM}} / d \ln a \gg d \ln M_{\mathrm{CDM}} / d \ln a$. Once the halo reaches $M_{200, \mathrm{WDM}} \approx M_{c}$, the halo is the approximate size of its counterpart in the CDM cosmology and $d \ln M_{200} / d \ln a$ drops to match the rate of the CDM halo. The WDM halo then begins to accrete mass as already-collapsed clumps in a quasi-hierarchical fashion. The CDM halos with masses $M_{200} \leq M_{c}$, in contrast, have corresponding WDM halos that never accrete mass in virialized clumps. The MAHs of such WDM halos are much smoother and accrete the bulk of their mass in a single period of rapid accretion.

Figure 9 shows ensemble average MAHs for halos from our large volume simulations. Here, the diamonds represent CDM halos and the crosses are WDM halos. Halos from different mass ranges have been offset in time to make the figure more readable. Both the CDM and WDM halos are selected to lie in the mass ranges $M_{200}=$ $(2-4) \times 10^{13} h^{-1} \mathrm{M}_{\odot},(0.5-1.3) \times 10^{14} h^{-1} \mathrm{M}_{\odot}$, and $>$ $4 \times 10^{14} h^{-1} \mathrm{M}_{\odot}$ at $a=100$. Figure 9 shows many of the same trends observed in Figure 8. For the most massive halos, the two cosmologies again show mass equality around $M_{200, \mathrm{CDM}} \approx M_{200, \mathrm{WDM}} \approx M_{c}$, with the WDM halos accreting mass significantly faster before this time. For $M_{200} \leq M_{c}$, WDM halos form later and more rapidly than CDM halos with similar mass. Also shown in the bottom panel of this figure are the growth rates, $d \ln \left(M_{200}\right) / d \ln (a)$, of the halos and their fits to equation (9).

Wechsler et al. (2002, hereafter W02) proposed a fitting formula for the MAH of a halo up to the present epoch of the form

$$
M(a)=M_{0} e^{-\left(a_{c, W 02} / a_{0}\right) S\left(a_{0} / a-1\right)} .
$$

The free parameter $S$ in this equation is used only in defining $a_{c, W 02}$, the creation epoch for the halo, when $d \ln M / d \ln a=S$. We choose to follow their convention and adopt $S=2$. This formula is fit to our CDM

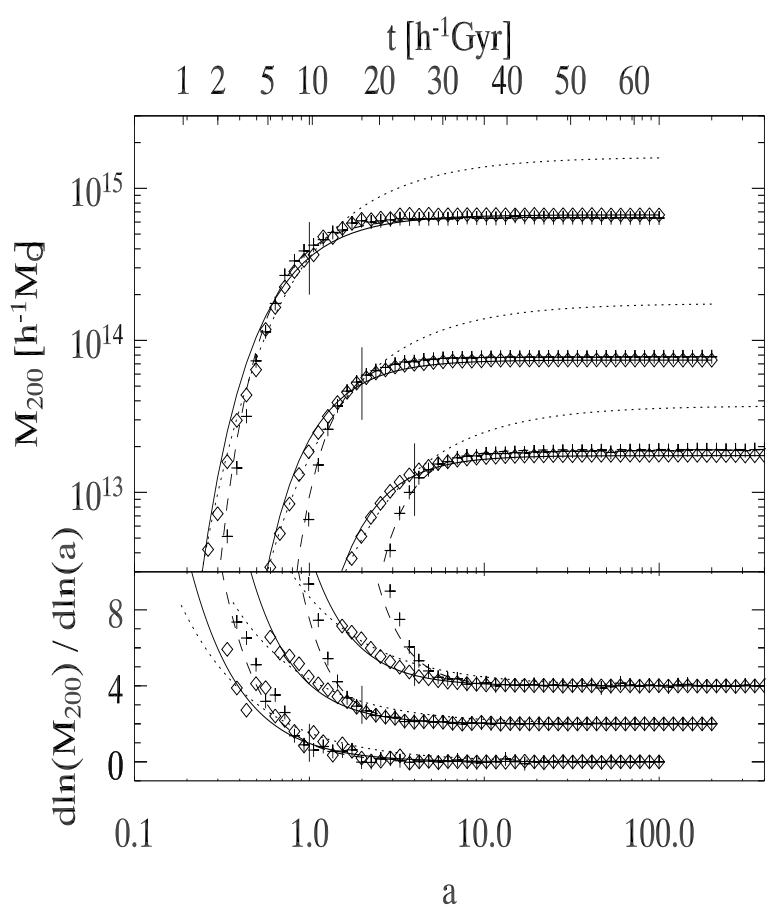

Fig. 9.- Top Panel: Average MAHs for CDM (diamonds) and WDM halos (plus symbols) from our large volume simulations. The halos are selected from the mass ranges $M_{200}=$ $(1-4) \times 10^{13},(0.5-1.3) \times 10^{14}$, and $>4 \times 10^{14} h^{-1} \mathrm{Mpc}$. The curves are offset in $a$ by a factor of 2 (intermediate mass range) and 4 (low mass range) to make them easier to distinguish, but the short vertical lines represent $a=1$ for all mass ranges. The dotted curves are fits to equations (8) for the CDM halos and the solid and dashed curves are fits to equation (9) for the CDM and WDM cosmologies. Bottom Panel: The mass growth rates, $d \ln \left(M_{200}\right) / d \ln (a)$, of the halos and plotted above and their fits to equation (9). Curves are offset in both the horizontal and vertical directions to make them more distinguishable.

MAHs over the range $a=0.2-1.0$ (but continued out to $a=100$ ) and plotted as the dotted curves in Figure9. In general, the fit is good for both CDM and WDM (not shown) models for $a<1$, but overestimates halo masses by a factor of 2 in the CDM run and more than an order of magnitude for the WDM run at late times. If the fit is calculated for the full range, $0.2<a<100$, the late time asymptote is correct, but $d \ln M_{200} / d \ln a$ is substantially lower than observed for either cosmology at all epochs. This behavior is probably an indirect result of the coincidence problem - the surprising observation that we live during the relatively short epoch where $\Omega_{M} \approx \Omega_{\Lambda}$. The fit works well for $a<1$, even when calculated for just a fraction of the region and then extrapolated. Although equation (8) was created for halos in a $\Lambda \mathrm{CDM}$ universe, $a=1$ is not much later than the equality epoch, $a_{e q}=0.75$, when $\Omega_{\mathrm{m}}=\Omega_{\Lambda}$. Once $\Lambda$ becomes the dominant component of the universe, the growth function quickly saturates and halos cease to grow (Busha et al. 2005). Consequently, equation (8) approaches its asymptote much more slowly than halos feeling the full effects of a dominant cosmological constant. To capture the full histories in both cosmologies, we propose a generalization of the form

$$
M(a)=M_{0} e^{-\left(a_{c} / a_{0}\right)^{\gamma} \frac{S}{\gamma}\left(\left(a_{0} / a\right)^{\gamma}-1\right)},
$$




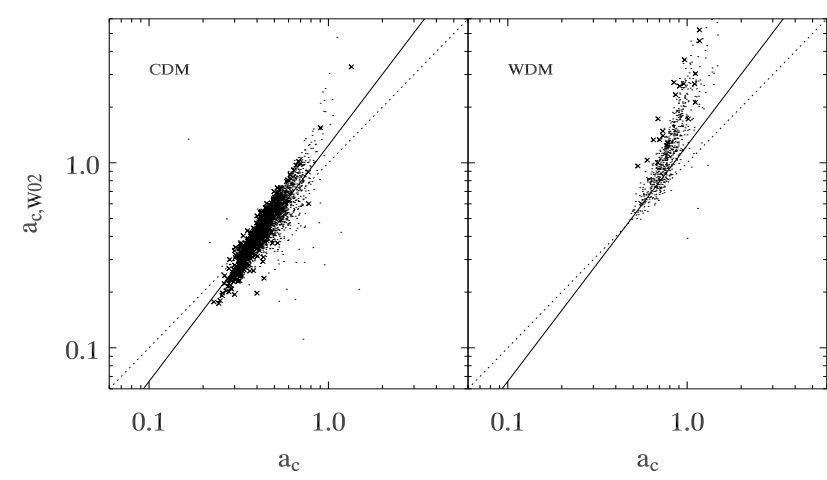

FIG. 10. - Comparison of the formation epochs defined by W02 (equation 8) and our generalization (equation [9]). The left panel represent halos from the CDM run and the right panel shows WDM halos. Dots are halos from the large volume run and crosses are from the small volume run. The dotted line shows an exact correspondence and the solid black line is a polynomial fit to the CDM halos.

where $\gamma$ is the rate index which sets the mass growth rate through the relation $d \ln M / d \ln a=S\left(a_{c} / a\right)^{\gamma}$. This variable is introduced such that $a_{c}$ is still the epoch where $M(a)$ has a logarithmic slope of $S$, but $\gamma$ sets how quickly a halo grows and asymptotes to its equilibrium mass. When $\gamma=1$ this generalized form reduces to equation (8). We expect to recover $\gamma>1$, which corresponds to more rapid formation and faster asymptote behavior (see Figure 9)

The fits to equation (9) in Figure 9 (solid and dashed curves for CDM and WDM halos) closely follow the measured MAHs. They provide good agreement over all epochs and work equally well for both CDM and WDM halos. Although not shown, the errors for this fit are generally $\lesssim 5-10 \%$, depending on the number of halos we average over. Figure 10 compares $a_{c, W 02}$, from equation (8), and $a_{c}$ from our modified form, equation (9), with dots representing halos from the large volume simulations, and crosses are from the small volume simulations. In the CDM cosmology (left panel), $a_{c, W 02}=2.7 a_{c}^{1.4}$ with a $27 \%$ scatter. The few WDM halos with $a_{c, W 02}<1$ are also well described by this relation. In both cases, there are several halos with $a_{c, W 02}>1$. These are typically halos that first appear around $a \geq 0.7$ and have rapid growth phases, powered either by major mergers or the collapse of a sub-truncation scale perturbation in the WDM cosmology. These formation epochs, based on equation (8), appear unphysical since they give formation epochs in the period of exponential expansion when halo growth has stopped. Equation (9) resolves this issue by increasing the halo rate index, resulting in a substantially lowered $a_{c}$ which pulls these halos significantly above the measured linear relation between $a_{c}$ and $a_{c, W 02}$.

Figure 11 plots the variation of $a_{c}$ (top panels) and $\gamma$ (bottom panels) with mass for CDM (left panels) and WDM (right panels) halos. Again, dots represent halos from the large volume realizations, and crosses are halos from the smaller volumes. Common to both the left and right panels are average trend lines for the CDM (solid curves) and WDM (dashed curves) halos. The plot shows all halos from our simulations that are well resolved at the end of the simulation $\left(M_{200}>400\right.$ particles for the large volume realizations and $M_{200}>1000$ particles for the smaller volume realizations - see ap- pendix for a further discussion). At the high mass end, $M_{200}>2 M_{c}=2 \times 10^{14} h^{-1} \mathrm{M}_{\odot}$, the average $a_{c}$ 's differ by only $\sim 10 \%$, less than the scatter for either cosmology. The rate index, $\gamma$, however, is about $50 \%$ higher in the WDM cosmologies at these high masses, reflecting the steeper MAHs presented in right side of Figure 9.

As mass decreases, both $a_{c}$ and $\gamma$ behave differently in the two cosmologies. The formation epoch decreases with mass in the CDM runs (in accordance with "bottom up" structure formation) but actually increases with mass in the WDM cosmologies. The number of WDM halos that exist with $M_{200} \ll M_{c}$ - all with later formation times than halos with mass greater than $M_{c}$ - again suggests that most small halos form through an instability of regions inside larger structures. This claim is consistent with our picture of sub-truncation scale halos forming through incomplete collapse of larger perturbations. For CDM halos, we again see the presence of the hierarchical structure formation from the fact that $\gamma$ is relatively constant throughout the entire mass range, $\gamma \approx 2$ with modest scatter. In contrast, the WDM cosmology has $\gamma$ increasing with lower masses, roughly as $\gamma \propto M_{200}^{-0.2}$. The spray of particles with $a_{c}$ and $\gamma$ much larger than the averages in the CDM cosmologies is a result of the difficulty in measuring these parameters for poorly resolved halos. The MAH cannot be measured accurately for halos that do not grow substantially above our resolution limit, and consequently our fit parameters have large uncertainties. The mean relation is actually within these uncertainties for all the low mass halos.

We also compare our modified MAH fitting formula with the model of W02 in the range of halo formation up to the present epoch in Figure [12. In this figure, we compare the residuals of fits of the average MAH to equation (9), fit from $a=0.3-100$ (dark line) and $a=$ $0.3-1$ (medium line) with equation (8) fit from $a=$ $0.3-1$ (light line) for different mass ranges taken from our CDM simulations. The RMS values for the residuals in $\ln M$ are shown in Table 3. While the rms residual between our modified fitting formula from $a=0.3-100$ is approximately a factor of 2 lower than the W02 model for all mass ranges, equation (9) fit from $a=0.3-1$ offers a substantial improvement over equation (8), decreasing the rms residual by a factor of 5 or more at the plotted masses. The introduction of $\gamma$ is apparently an important correction for the early time MAH growth as well as the late time asymptote. Table 4 lists the values for $a_{c}$ and $\gamma$ for the profiles of Figure 12. The fits are robust in the sense that $a_{c}$ does not change substantially depending on how the MAH is calculated. In all cases, however, the best fit returns $\gamma$ substantially larger than 1, and may have some mass dependence. The exact value of $\gamma$ depends strongly on the fit range, with fits out to $a=$ 100 requiring a larger value in order for the MAH to asymptote properly. These larger values of $\gamma$ in turn push $a_{c}$ slightly earlier. Fitting in the range $0.3 \leq a \leq 1$, results in $\gamma \approx 1.6$ with values for $a_{c}$ that differ from those of the W02 model by only $4 \%$. While our average halo MAHs all appear to have $\gamma>1$, there is a much larger spread when considering fits for individual halos. Here, $\gamma$ ranges from $0.2-10$ and the fits have residuals that are typically $20 \%$ lower than those of equation (8). 


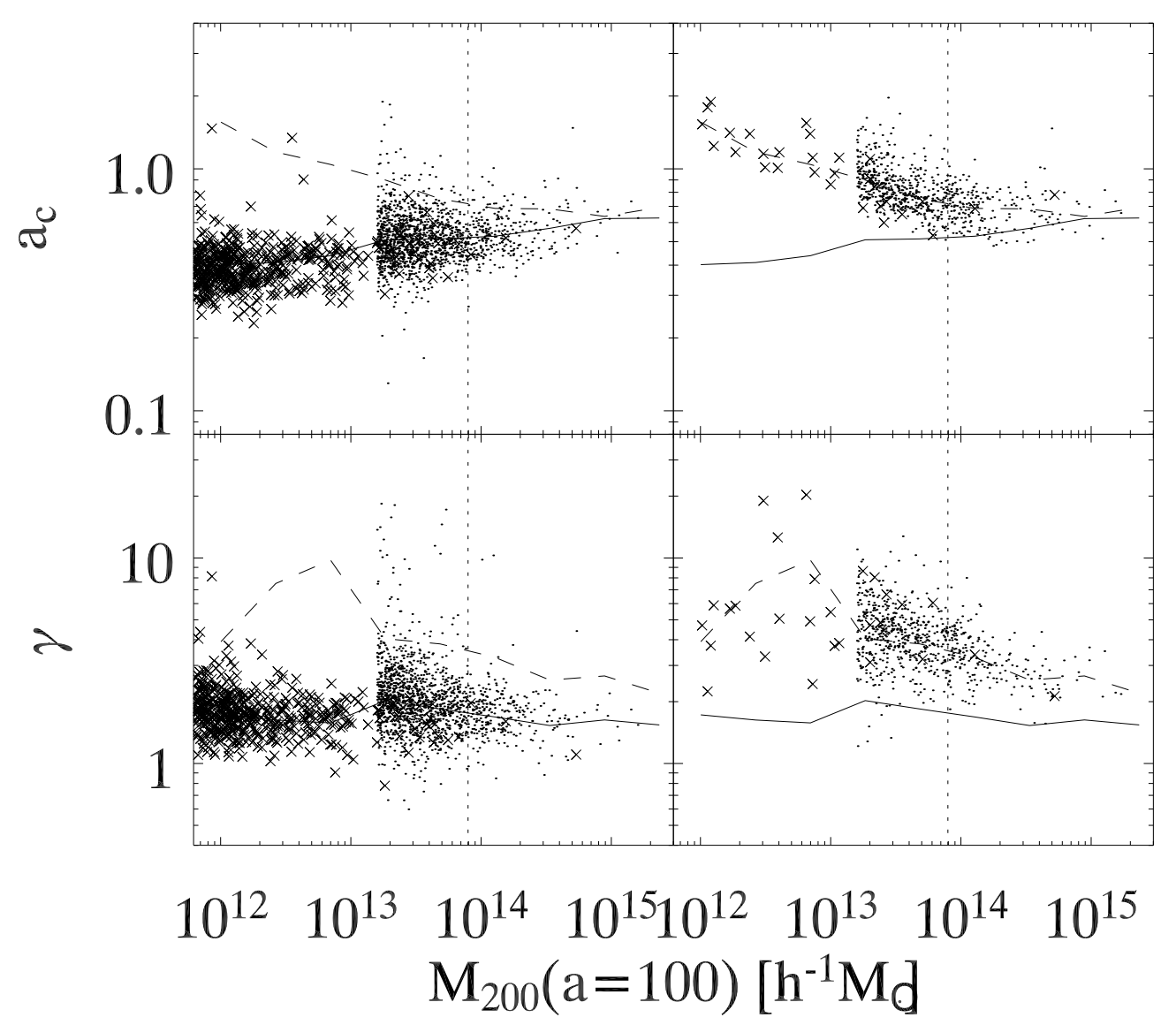

Fig. 11. - Top Panels: The dependence of halo's formation epoch ( $a_{c}$ in equation 9 $)$ on mass. The left panel shows halos from the CDM run, and the right halos from the WDM cosmology. Dots represent halos from the large box run, and crosses are from the small box run. The solid and dashed curves show the trend of $a_{c}$ mass for the CDM and WDM cosmologies, and the vertical dotted line is $M_{c}$. Bottom Panel: Same as the top panel, but now plotting the behavior of $\gamma$, the amplification factor of equation (9), as a function of mass.

TABLE 3

RMS RESIDUALS OF MAH FITS:

\begin{tabular}{cccc}
\hline$M_{200}\left[h^{-1} \mathrm{M}_{\odot}\right]$ & $\begin{array}{c}\text { Equation (9) } \\
a=0.3-100\end{array}$ & $\begin{array}{c}\text { Equation (9) } \\
a=0.3-1\end{array}$ & $\begin{array}{c}\text { Equation (8) } \\
a=0.3-1\end{array}$ \\
\hline$(0.6-1.2) \times 10^{14}$ & 0.14 & 0.044 & 0.21 \\
$(3-6) \times 10^{13}$ & 0.14 & 0.036 & 0.31 \\
$(1.5-3) \times 10^{13}$ & 0.15 & 0.088 & 0.43 \\
\hline
\end{tabular}

\subsection{Halo Substructure}

In this section, we compare the subhalo distribution of our CDM and WDM cosmologies, considering only halos from our smaller volume simulations. While these simulations do not contain a statistically large number of halos, the larger volume simulations do not have the necessary resolution to accurately describe the subhalo population.

Not surprisingly, the most dramatic difference between the subhalo populations of our CDM and WDM halos is their abundance. In the CDM simulation, the average number of subhalos with $M>1.24 \times 10^{10} h^{-1} \mathrm{M}_{\odot}$ (20 particles) is roughly proportional to the mass of the host halo, $\bar{n}_{\text {subs }} \propto M_{200}$ at $a=1$. For host halos of all masses, approximately $10 \%$ of the host mass is in bound substructures at this epoch, a value consistent with previous studies (Klypin et al. 1999). In contrast, for the WDM cosmology $\bar{n}_{\text {subs }} \propto M_{200}^{0.4}$, with only about $5 \%$ of the host mass in bound subhalos. By $a=100$ in the CDM cosmology, the slope of the number of subhalos with mass has not changed substantially, $\bar{n}_{\text {subs }} \propto M_{200}^{1.2}$, but many subhalos have been destroyed, and only $0.3 \%$ of the mass of an average halo is contained in substructure. The steepening of the slope is caused by a more efficient destruction of subhalos in hosts with lower masses. Small halos today contain smaller, more weakly bound, subhalos than larger hosts. Additionally, these small halos undergo fewer future mergers to replenish their subhalo population. By contrast, with the exception of the single largest WDM halo, none of the WDM halos contain any substructures at $a=100$. Even this largest halo has only $\bar{n}_{\text {subs }}=2$, as opposed to $\bar{n}_{\text {subs }}=137$ in its CDM counterpart. Surprisingly, the shape of the subhalo mass function, $d n_{\text {sub }}\left(M_{\text {sub }}\right) / d \ln \left(M_{\text {sub }} / M_{\text {Host }}\right)$, does not change substantially between today and $a=$ 100. The mass function is truncated at the high mass end $\left(M_{\text {sub }} / M_{\text {Host }}>0.01\right)$ and is slightly steepened. This is due to the increased effect of dynamical friction on more 
TABLE 4

MAH PARAMETERS:

\begin{tabular}{cccccc}
\hline$M_{200}\left[h^{-1} \mathrm{M}_{\odot}\right]$ & $a_{c, W 02}$ & $a_{c}$ & $a_{c}$ & $\gamma$ & $\gamma$ \\
& $0.3-100$ & $0.3-1$ & $0.3-1$ & $0.3-100$ & $0.3-1$ \\
\hline$(0.6-1.2) \times 10^{14}$ & 0.54 & 0.47 & 0.52 & 2.0 & 1.5 \\
$(3-6) \times 10^{13}$ & 0.53 & 0.50 & 0.52 & 2.1 & 1.6 \\
$(1.5-3) \times 10^{13}$ & 0.48 & 0.48 & 0.50 & 2.3 & 1.8 \\
\hline
\end{tabular}

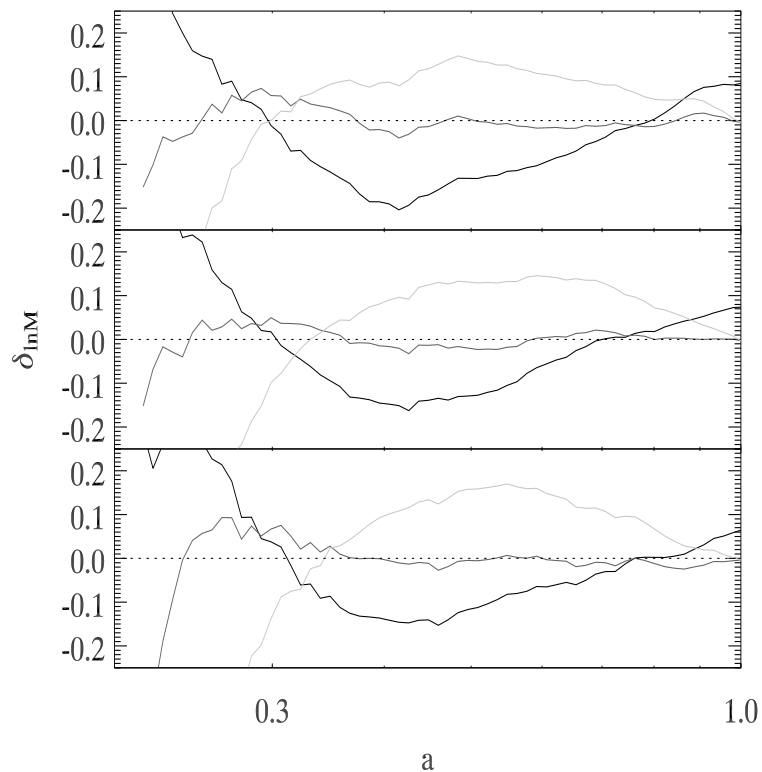

$\mathrm{a}$

Fig. 12.- Residuals of average MAHs to various fits. The dark lines are the residuals to equation (9) fit in the range $a=0.3-100$. The medium lines also use equation (9), but the fit is calculated over $a=0.3-1$. The light lines fit the model of Wechsler et al. (2002), equation (8), to the range $a=0.3-1$. The halos are in the mass ranges (top to bottom) $M_{200}=(0.6-1.2) \times 10^{14},(3-6) \times$ $10^{13},(1.3-3) \times 10^{13} h^{-1} \mathrm{M}_{\odot}$.

massive objects, pulling them towards the center of the halo where they are more easily disrupted and stripped of mass.

Figure 13 shows the evolution of the average number of subhalos in halos of various masses. CDM and WDM halos of all sizes show similar evolution in the average number of subhalos. For $a \leq 3$, mergers create substructure, resulting in an increasing $\bar{n}_{\text {subs }}(a)$. After mergers end, $a \approx 3$, no new subhalos are accreted and existing subhalos gradually fall inward and are disrupted due to dynamical friction and tidal forces. The number of subhalos in the WDM cosmology, however, drops much more rapidly than in the CDM cosmology. The primary reason for this difference is the lower binding energy of the subhalos caused by the later formation epoch of lowmass WDM halos. When these halos accrete onto more massive halos, they are much more prone to disruption and consequently have a shorter life. While WDM subhalos are formed with a lower binding energy/density, most other properties, including their average velocity, velocity dispersion, and mass, differ very little between the CDM and WDM models. The only other systematic

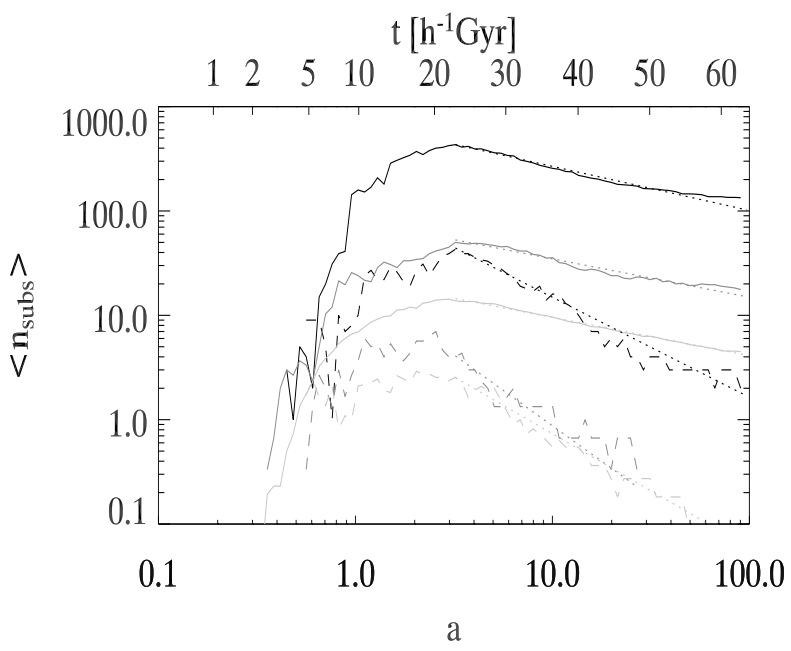

FIG. 13.- The evolution of the average number of subhalos in host halos of various masses. The solid curves indicate CDM halos, and the dashed WDM halos. At $a=100$, the halos have masses $M_{200}=(2-4) \times 10^{13}$ (light line), $(0.5-1.3) \times$ $10^{14}$ (medium line), and $>4 \times 10^{14} h^{-1} \mathrm{M}_{\odot}($ dark line $)$. The dotted lines show fits to equation (10).

difference is the average distance a subhalo lives from the center of its host - WDM subhalos tend to live further out. This is also related to the lower binding energy of WDM subhalos, since they are more easily disrupted when they move closer to center of their host. As a measure of subhalo destruction, we have fit the evolution of $\bar{n}_{\text {sub }}$ during the late-time deSitter expansion (when the host halos are no longer being disrupted by mergers) to an exponential decay,

$$
\bar{n}_{s u b}(a)=\bar{n}_{m} e^{-\alpha^{\prime}\left(t-t_{m}\right)}=\bar{n}_{m}\left(a / a_{m}\right)^{-\alpha},
$$

where the subscript $m$ denotes the epoch where the subhalo population is at its maximum, and $\alpha^{\prime}$ is the subhalo decay rate, with $\alpha^{\prime}=H \alpha=\alpha 0.0856 \mathrm{Gyr}^{-1} \mathrm{~h}$ for our $\Lambda$ dominated cosmology $\left(H=H_{\infty}\right)$. For our CDM halos, we get $\alpha=0.38 \pm 0.03$, while for the WDM halos there is a much more rapid decay, $\alpha=1.1 \pm 0.2$, yielding subhalo half-lives of $21 \pm 2$ and $7.4 \pm 1 h^{-1} \mathrm{Gyr}$, respectively. Figure 13 makes it appear that equation (10) fits better for lower mass halos. This, however, is an artifact of the fact that we have only a few high mass halos in each simulation, giving us poor statistics.

\subsection{Density Profile}

The radial density profile is one of the most fundamental halo properties. Previous WDM studies 


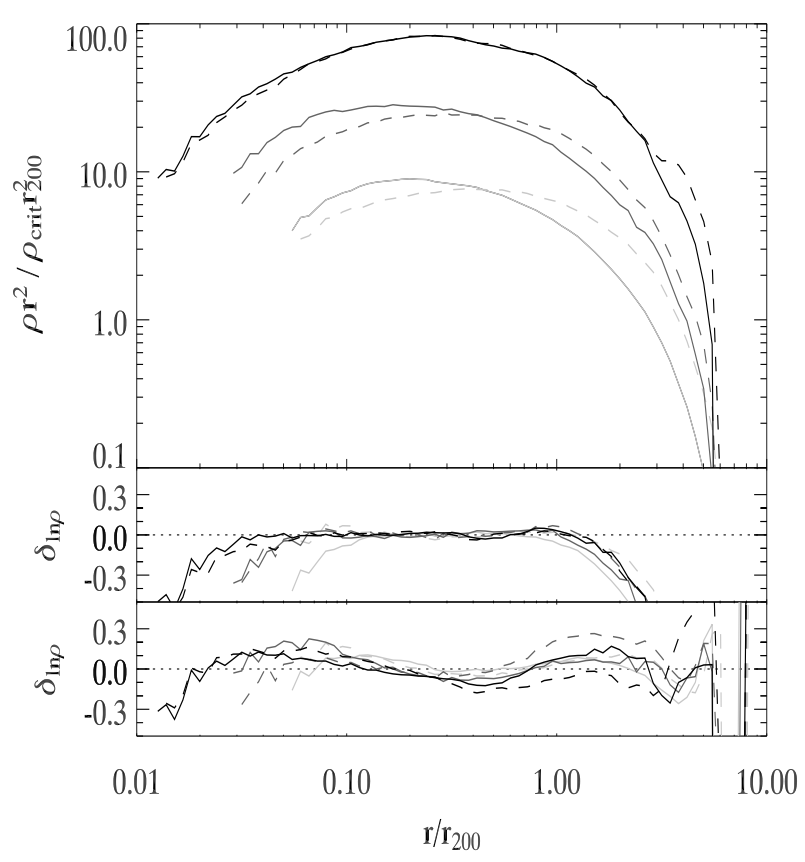

FIg. 14.- Top Panel: Average density profiles times $r^{2}$ for all bound material in halos at $a=100$ from our smaller volume runs. Halos selected according to the mass ranges $M_{200}=$ $(2-4) \times 10^{13}$ (light line),$(0.6-1.3) \times 10^{14}$ (medium line) , and $>$ $4 \times 10^{14} h^{-1} \mathrm{M}_{\odot}$ (dark line), with the solid and dashed lines representing CDM and WDM halos. The profiles have been offset from each other to make them easier to see. Middle Panel: Residuals of NFW fits to the above six profiles. Bottom Panel: Residuals of truncated Hernquist fits (equation [11) to the above six profiles.

(Moore et al. 1999; Colín, Avila-Reese, \& Valenzuela 2000; Bode. Ostriker. \& Turok 2001) indicate that WDM halo density profiles do not differ substantially from their CDM counterparts, and our simulations support this finding. Figure 14 shows average density profiles for all particles bound to halos from the CDM and WDM cosmologies at $a=100$ from our smaller volume runs. The solid lines are CDM halos from the mass ranges of Figure 9, and the dashed lines are WDM halos. The different mass ranges have been offset and the density multiplied by $r^{2}$ to make the differences between the various profiles more visible. The halos can be described by an NFW profile, equation(5) for the range $0.05 r_{200} \lesssim r \lesssim r_{200}$. Here, $r_{s}$ and $\rho_{s}$ are the the NFW scale radius and density. The middle panel of Figure 14 shows the residuals to the NFW fits for the nine plotted profiles, which generally fall in the $\sim 5 \%$ range.

Previous work (Busha et al. 2005) has shown that well before $a=100$, halos in a $\Lambda$-dominated universe develop clear edges at $r \approx 4.6 r_{200}$. Beyond $r_{200}$, the NFW profile is much too shallow and the density profile for all bound material is better fit by a truncated Hernquist profile,

$$
\rho=\frac{\rho_{0}}{\left(r / r_{c}\right)\left(1+r / r_{c}\right)^{3}} e^{\left(-r / r_{\text {halo }}\right)^{5.6}},
$$

where $\rho_{0}$ and $r_{c}$ are the central density and core radius, and $r_{\text {halo }}$ is the radial extent of all bound halo material. Residuals to this fit are shown in the bottom panel of Figure 14 This fit has larger residuals than NFW for $r<r_{200}$, but has much smaller residuals at larger radii

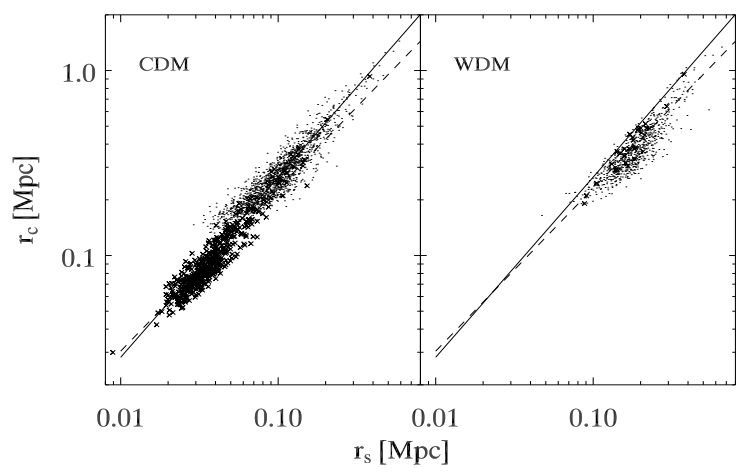

FIG. 15. - Comparison of the core radius, $r_{c}$, of the Hernquist fit (equation [11) plotted against the scale radius, $r_{s}$, of the NFW fit (equation 5) from our CDM and WDM simulations. Dots represent halos from the large box run, and crosses are from the small box run. The black and dashed lines show the linear fits for the CDM and WDM cosmologies.

and provides a good description of the halo out to its actual edge.

As with the MAHs, there is little difference between the density profiles of the WDM and CDM cosmologies for halos with $M_{200} \gg M_{c}$. Specifically, for the plotted profiles in this range, most differences are at the $\sim 1 \%$ level. Significant differences appear as we near the truncation scale, $M_{c}$, but, remarkably, the profiles are still well fit by both the NFW and Hernquist profiles, as indicated by the residuals in the bottom panels of Figure 14. We define the concentration in the usual way, $c_{200}=r_{200} / r_{s}$. As noted above, the parameter $r_{s}$ is measured by fitting an NFW profile to our profiles using logarithmically spaced radial bins in the range $0.05 r_{200}-r_{200}$ (similar to Bullock et al. 2001). For equilibrated halos at $a=100$, the steepness of the halo density profile causes $r_{s}$ to change substantially if we fit to a different radius. By increasing the outer fit radius to $r_{v i r} \approx 2.5 r_{200}$ as in Bullock et al. (2001), concentrations typically decrease by a factor of 2 or greater, depending on the mass of the halo. A more robust concentration could alternatively be measured as $c_{\text {halo }}=r_{\text {halo }} / r_{c}$ from equation (11), although the definition using the NFW fit is more standard (and our new definition is not well defined for $a \lesssim 3$ ). Figure 15 plots the relation between the parameters $r_{c}$ and $r_{s}$. The relation is well fit by a power law, $r_{c} \propto r_{s}^{\nu}$, with $\nu=0.974 \pm 0.004,0.88 \pm 0.02$ and proportionality constants 0.40 and 0.24 for the CDM and WDM cosmologies. For what follows, however, we will continue to use the concentration defined with an NFW fit.

Figure 16 shows the dependence of the concentration on mass for our halos. As with Figure 11] the left panel shows CDM halos and the right panel WDM halos, with dots representing halos from the large volume realizations and crosses halos from the small volume realizations. This plot follows many of the overall trends of Figure 11, Again, for $M_{200} \gg M_{c}$ the average values for $c_{200}$ differ by less than $10 \%$ for CDM and WDM halos and systematically diverge for lower masses. The low mass WDM halos are much "puffier" than similar mass halos (or their counterparts) in the CDM simulations. W02 explained the trend of changing $c_{200}$ with $M_{200}$ by identifying a relationship between $c_{200}$ and $a_{c}$, which we have 
reproduced with our data in Figure 17. Both the CDM (left panel) and WDM (right panel) halos follow a power law relation $c_{200} \propto a_{c}^{-\beta}$, although there is a substantial amount of scatter present in the CDM relation. For CDM halos, $\beta=0.79 \pm 0.2$ (solid line), while the WDM halos have $\beta=0.86 \pm 0.3$ (dashed line), values that are both within $2 \sigma$ from the combined slope $\beta=0.79 \pm 0.1$ (dotted line). This finding is similar to the $c \propto a_{c}^{-1}$ relation proposed by W02. Note that for W02, $r_{200}$ is replaced with $r_{v i r} \approx 2.5 r_{200}$ at a $=100$ (Eke. Cole. \& Frenk 1996). The explanation for this trend is that $c_{200}$ is a reflection of the average density at the time of collapse, so that halos forming earlier should have higher concentrations, exactly as observed. This trend holds even for WDM halos with $M_{200} \ll M_{c}$. This picture is further enforced by a toy model for concentrations proposed by Eke. Navarro, \& Steinmetz (2001), modified to use our density threshold. Noting that in WDM models $c_{200}$ decreases with mass below the truncation scale, Eke et al. (2001) defined an effective perturbation spectrum,

$$
\sigma_{e f f}(M)=-\frac{\mathrm{d} \sigma(M)}{\mathrm{d} \ln (M)}
$$

so that the spectrum also decreases at low masses. From this equation, a collapse epoch, $a_{c \text {,toy }}$ can be identified as the epoch where $D\left(a_{c, \text { toy }}\right) \sigma_{e f f}\left(M_{s}\right)=1 / C_{\sigma}$, where $M_{s}=M\left(<2.17 r_{s}\right)$ (the mass contained within the radius where the circular velocity of a NFW profile reaches its maximum) and $C_{\sigma}$ is a fitting parameter. A central density is defined as in Bullock et al. (2001) such that $M_{200}=4 / 3 \pi r_{s}^{3} \tilde{\rho}_{s}$. We then assume that

$$
\tilde{\rho}_{s}=200 \rho_{\text {crit }}\left(a_{0}\right) c_{200}^{3}
$$

and set this density scale equal to our overdensity at the epoch of formation, $200 \rho_{c r i t}\left(a_{c, \text { toy }}\right)$. This procedure yields the relation

$$
c_{200}=\left[\frac{\rho_{\text {crit }}\left(a_{c, \text { toy }}\right)}{\rho_{\text {crit }}\left(a_{0}\right)}\right]^{1 / 3} .
$$

The only free parameter in this model is the constant $C_{\sigma}$, which we set equal to 32 . The results of this model are plotted as the lighter curves in Figure 11, The model characterizes the simulated halos for both the CDM and WDM cosmologies at $a=100$, and produces equally good agreement at earlier epochs. Our toy model differs from that of Eke et al. (2001) only in that we define our halos in terms of $200 \rho_{\text {crit }}$, as opposed to the epoch-dependent quantity $\Delta(a) \rho_{\text {crit }}$. We find that using $\Delta(a) \rho_{\text {crit }}$ requires different values for $C_{\langle\sigma v\rangle}$ at $a=1$ and 100.

In all of our simulations, the density profile is well characterized by an NFW profile for much of the halo's radial extent, and that the quality of the fit does not depend on whether a halo was taken from a CDM or WDM cosmology. The primary difference between halos in these two models - the change in concentration parameter - follows a simple relationship with the formation epoch that is relatively insensitive to how the halos form: CDM halos that have accreted their mass as virialized clumps; WDM halos with $M_{200}>M_{c}$ that have formed through a period of rapid smooth accretion followed by growth through accretion of clumps; and WDM halos with $M_{200}<M_{c}$ that have formed through a single period of rapid smooth accretion.

\section{CONCLUSIONS}

This work examines the effects of small scale structure and merger activity on the formation and ultimate structure of halos in a $\Lambda$-dominated universe. Using $\mathrm{N}$ body simulations with an initial power spectrum that is truncated on small scales, we model a WDM-like universe where the formation of early low-mass objects is suppressed and compare the results to standard $\Lambda \mathrm{CDM}$ simulations. Without these seeds for hierarchical growth, halos above the truncation scale form through an initial rapid accretion phase, resulting in objects with mass $M_{200} \sim M_{c}=8 \times 10^{13} h^{-1} \mathrm{M}_{\odot}$. These objects can then grow through mergers with other large halos. Many halos also form below the truncation scale through incomplete collapse of larger perturbations. These halos form solely through a monolithic-like collapse process. Regardless of their size, WDM halos typically form later and faster, with a larger $\dot{M}_{200}(a)$ than their counterparts in an $\Lambda$ CDM cosmology.

To describe this rapid accretion, we generalize the Wechsler et al. (2002) mass accretion history formula by introducing a rate index, $\gamma$, that controls the growth rate evolution, $d \ln (M) / d \ln (a) \propto a^{-\gamma}$. This parameter is necessary in order to fit the halo MAH into the far future. Otherwise, the halo mass approaches its asymptote much too slowly. Additionally, for ensemble-averaged halo histories fit to the present epoch $(a \leq 1)$, we recover $\gamma \sim 1.6$, substantially higher than the $\gamma=1$ assumed in Wechsler et al. (2002). The larger $\gamma$ improves the mean MAH fit by a factor of 5 . Our generalization also reduces the number of objects that have unphysical formations epochs, $a_{c} \gg 1$.

We have also calculated abundances and decay rates for substructure in our dark matter halos. As expected, WDM halos contain much less substructure, and most host halos destroy all of their subhalos by the end of our simulations at $a=100$. The decay of subhalos is (on average) exponential in time with half-lives $21 \mathrm{Gyr}$ and 7.4Gyr for the CDM and WDM cosmologies. This is consistent with the picture discussed below where later formation times create lower concentration objects that are more prone to disruption.

Despite differences in the formation process and substructure abundance, WDM halos exhibit NFW density profiles, just like CDM halos, albeit with a different $c_{200}\left(M_{200}\right)$ relationship. Halos in WDM cosmologies have lower concentrations than their CDM counterparts, but follow the same $c_{200}\left(a_{c}\right)$ relation, allowing us to relate concentration to mass in both cosmologies using a 1 parameter toy model that characterizes the concentration using the linear power spectrum. This characterization is motivated by the idea that the concentration is set by the cosmological background density at the epoch of collapse. The form of the halo density profile persists, even though the method of formation and amount of substructure is changed substantially in our WDM cosmology. Taken together, our results suggest that the form of the halo density profile is set not by merger activity, but instead through large-scale modes of the gravitational relaxation process. Halos appear to be very efficient at erasing their initial conditions and do not care whether their mass was 


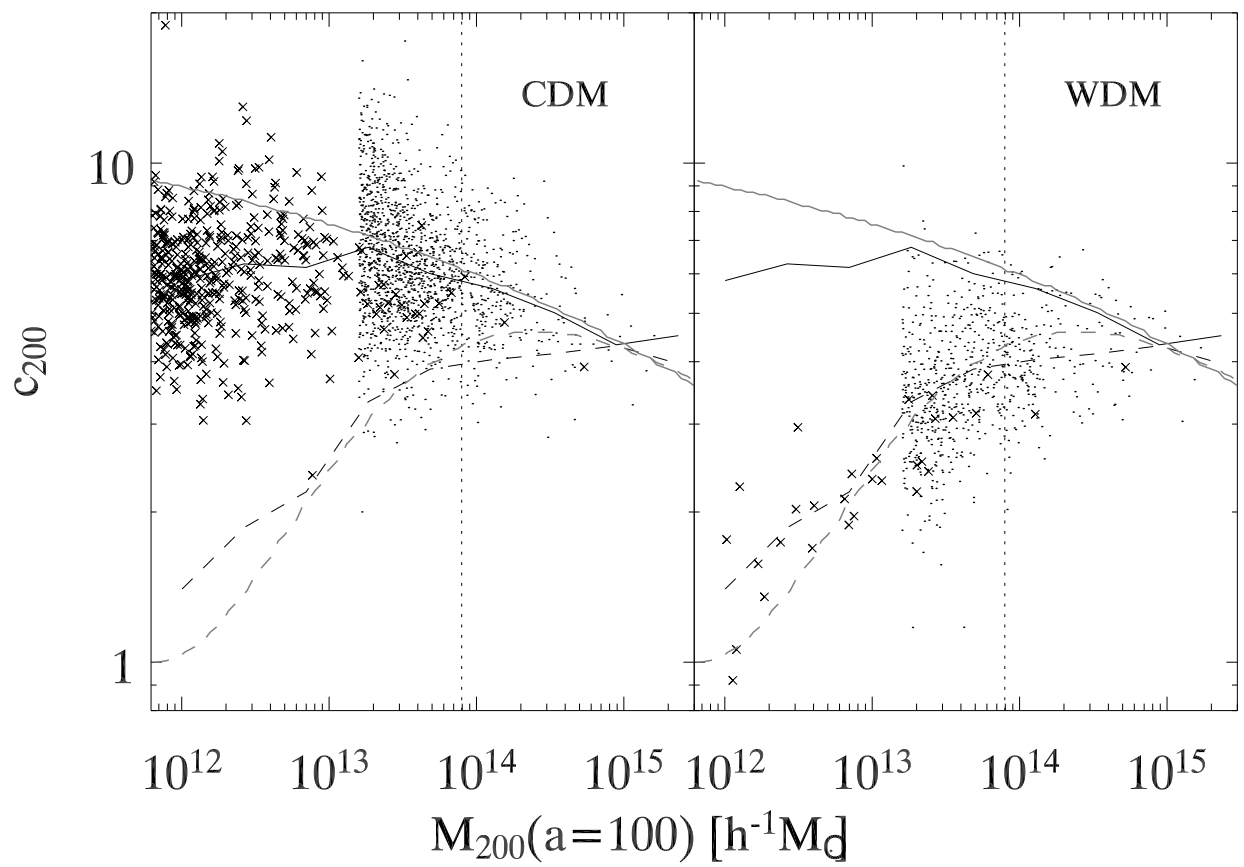

FIG. 16. - The concentration $\left(c_{200}=r_{s} / r_{200}\right)$ as a function of mass for CDM and WDM halos. Dots represent halos from the large box run, and crosses are from the small box run. The dark solid and dashed lines show average $c_{200}\left(M_{200}\right)$ values for CDM and WDM halos, and the vertical dotted line marks the truncation scale, $M_{c}$. The light solid and dashed lines show the calculated $c_{200}\left(M_{200}\right)$ relation from the toy model discussed in $\S 4.3$.

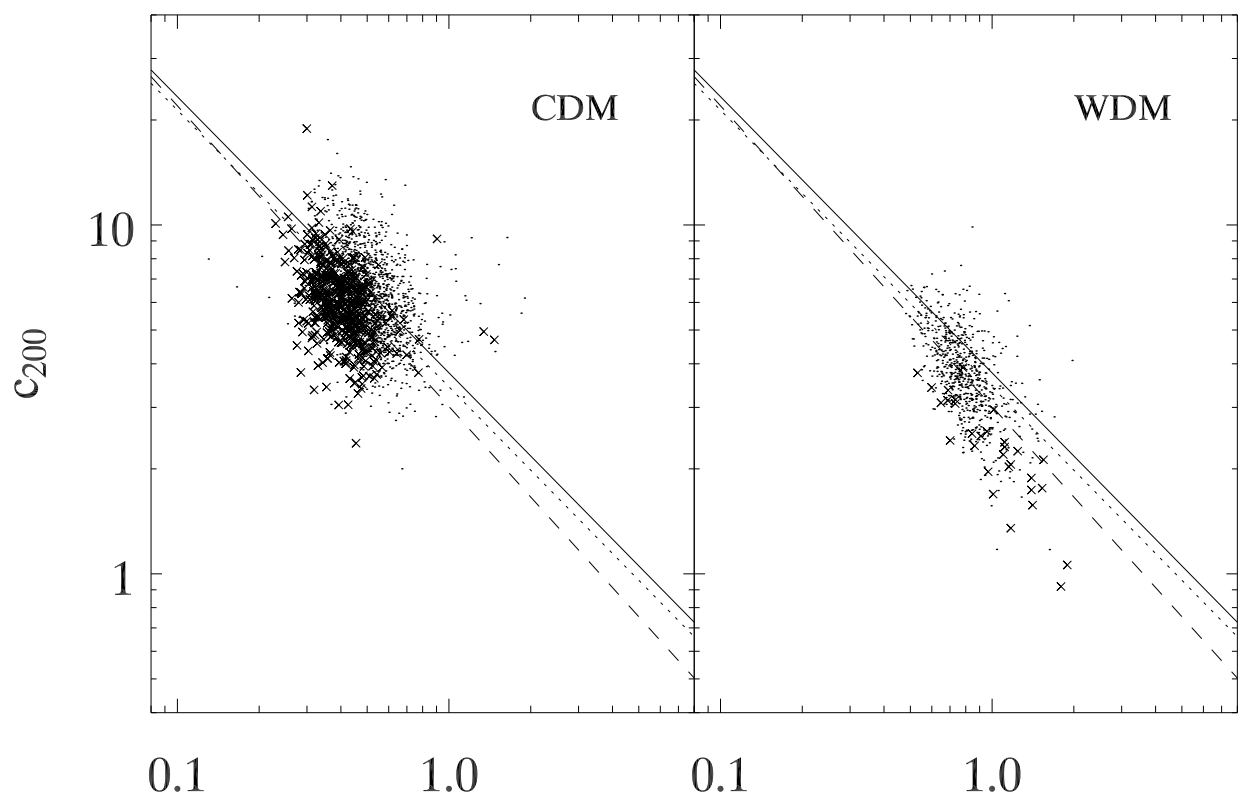

$\mathrm{a}_{\mathrm{c}}$

$\mathrm{a}_{\mathrm{c}}$

FIG. 17.- The concentration as a function of formation epoch, $a_{c}$ from equation (9) for CDM and WDM halos. Dots represent halos from the large box run, and crosses are from the small box run. The solid line is the trend-line for CDM halos, and the dashed line is the trend-line for WDM halos. The dotted line is the trend-line for the combined sample of all CDM and WDM halos. 


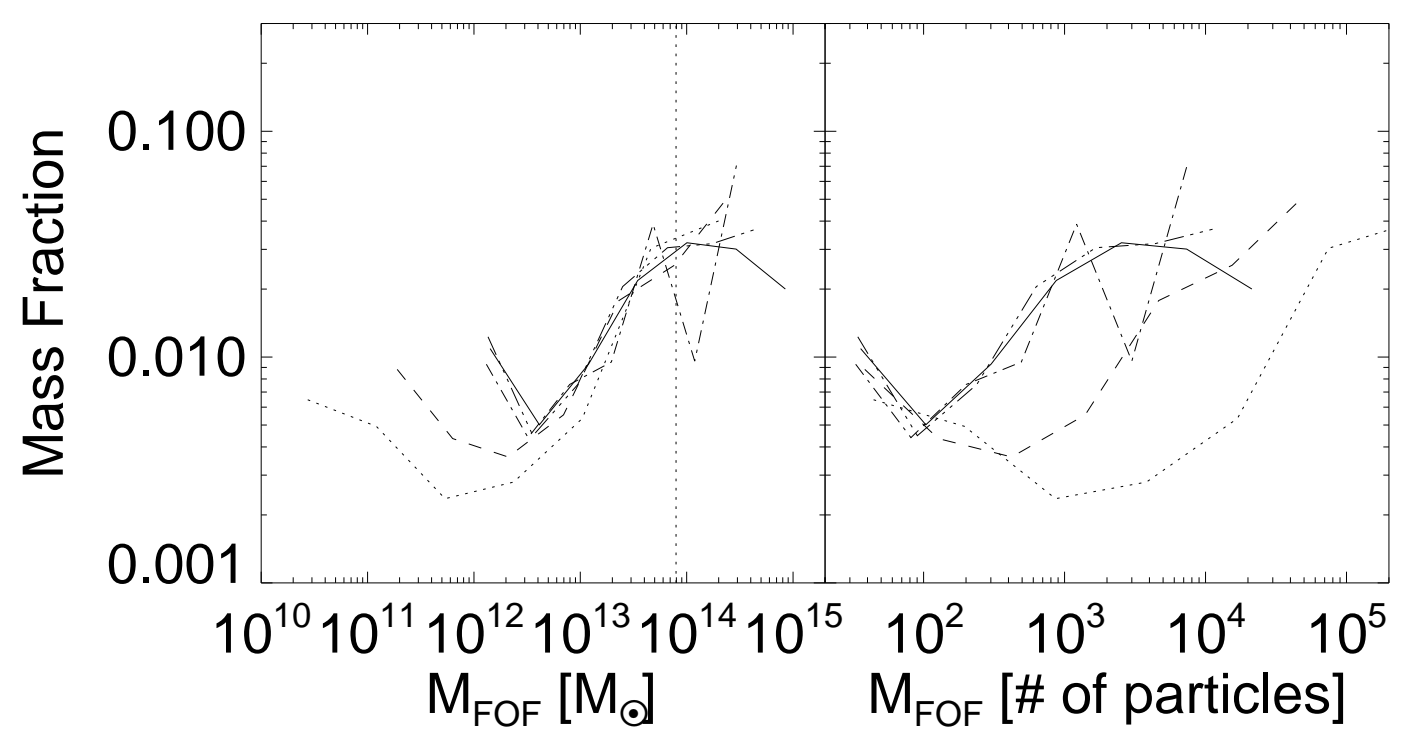

FIG. 18.- Left Panel: The fraction of mass in a collapsed structure of a given mass as a function of mass for a number of WDM simulations with varying degrees of mass particle resolution $\left(M_{\text {particle }}=6.20 \times 10^{8}-3.97 \times 10^{10} h^{-1} \mathrm{M}_{\odot}\right)$ and simulated volume $\left(50-200 h^{-1} \mathrm{Mpc}\right)$. The vertical dotted line represents the truncation scale, $M_{c}$. Right Panel: The same mass fractions, now plotted mass in units of number of particles.

accreted rapidly, slowly, in clumps, or continuously. The only aspect of the density profile directly linked to the halo formation process appears to be the concentration, which set by the formation epoch, the epoch when the mass accretion rate drops to a specific value. In particular, substructures and major mergers seem to have little effect in driving the equilibrium structure of a halo.

The authors would like to thank Volker Springel for the generous use of his SUBFIND routine and for useful discussion. This work has been supported at the Uni- versity of Michigan by the Michigan Center for Theoretical Physics, by NASA grants NAG5-13378 and NNG04GK56G0, NFS ITR grant ACI-0121671, and by the Foundational Questions Institute via Grant RFP106-1. M. T. B. was supported by a Predoctoral Fellowship from the Rackham Graduate School at the University of Michigan. A. E. E. acknowledges support from the Mill Foundation for Basic Research in Science at University of California, Berkeley.

\section{APPENDIX}

\section{MASS FUNCTION}

The upturn in the FOF mass function (Figure 6) at small mass is a surprising and potentially troubling feature of our WDM simulations, and it is necessary to determine if this phenomenon is a result of the simulation or of the FOF group-finding algorithm. We have conducted a series of 5 WDM simulations with various volumes and mass resolutions to examine this effect, and present the resultant mass fractions at $a=1$ in Figure 18. Here, we are plotting the mass fractions, as opposed to the mass functions shown in Figure 6. The mass fraction and mass function are related by $d n(M) / d \ln (M)=(\bar{\rho} / M) f(M)$. We used a mass resolution of $3.97 \times 10^{10} h^{-1} \mathrm{M}_{\odot}$ as our base model, conducting three runs at this scale with comoving box lengths of 200, 100, and $50 h^{-1} \mathrm{Mpc}$ (solid, dash-dot, and dash-dot-dot-dot lines), and two additional runs with box length $50 h^{-1} \mathrm{Mpc}$ with mass resolutions $6.20 \times 10^{8}$ and $4.96 \times 10^{9} h^{-1} \mathrm{M}_{\odot}(\mathrm{dotted}$ and dashed lines). The left panel shows the mass fraction as a function of mass in units of $h^{-1} \mathrm{M}_{\odot}$, while the right panel shows the same fractions in units of number of particles.

The most immediate feature of the left panel is that the location of the upturn changes with mass resolution, independent of the simulated volume. The right panel of Figure 18 further indicates that this upturn is a purely numerical artifact. Here, the mass fraction is plotted in units of number of particles in the halo, and we see that at low masses all resolutions converge on the upturn. Finally, we also note that for the two runs that have corresponding CDM cosmologies (solid and dotted lines) the location of the minimum in the mass fraction $\left(M_{\text {min }}\right)$ corresponds to a transition point: halos with $M>M_{\text {min }}$ have corresponding halos in the CDM simulation, while those with $M<M_{m i n}$ do not (see Figure 7). In these, $90 \%$ of all halos with $M_{F O F} \gg M_{\min }$ have corresponding halos in the CDM cosmology, while less than $1 \%$ of halos with $M_{F O F}<M_{\min }$ have identified cross halos. Even though the input power spectrum for the CDM and WDM cosmologies is vastly different, it is not the value of $M_{c}$, but rather the of value $M_{\text {min }}$ that determines the mass where we are unable to find corresponding halos in the CDM cosmology. This trend is especially noteworthy in the case of the small volume realization, where $M_{\min }$ is almost two orders of magnitude below $M_{c}$. Finally, the upturn appears substantially less pronounced for the SO mass function. Most of the FOF halos below the upturn have no central overdensity greater than $200 \rho_{\text {crit }}$ but instead exist as a more linear string of particles that are 
not even identified as energetically bound by SUBFIND .

One expects an upturn eventually, since a FOF group finder puts every particle in a group of some size. At any given time, 50-70\% of all particles end up in "groups" of one particle, requiring an upturn somewhere, something that you would expect even in a standard $\Lambda \mathrm{CDM}$ simulation (although the effect there should be much smaller due to the large number of actual groups at this mass range). Our results suggest that this contamination is an issue for groups containing fewer than

$$
N_{\text {min }} \approx 250\left(\frac{M_{\text {particle }}}{10^{10} \mathrm{M}_{\odot} h^{-1}}\right)^{-0.56}
$$

particles when a linking length of 0.164 is used.

\section{REFERENCES}

Aarseth, S. J., Turner, E. L., \& Gott, J. R., III 1979, ApJ, 228, $664 \mathrm{~A}$

Adams, F. C., \& Laughlin, G. 1997, RvMP, 69, 337

Avila-Reese, V., Cloín, P., Valenzula, O., D’Onghia, E., \& Firmani, C. 2001, ApJ, 599, 516

Bardeen, J. M., Bond, J. R., Kaiser, N., \& Szalay, A. S. 1986, ApJ, 304, 15

Binney, J., \& Tremaine, S. 1987, Galactic Dynamics (Princeton: Princeton University Press)

Blumenthal, G. R., Faber, S. M., Primack, J. R., \& Rees, M. J. 1984, Nature, 311, 517

Bode, P., Ostriker, J. P., \& Turok, N. 2001, ApJ, 556, 93

Bond, J. R., \& Szalay, A. S. 1983, ApJ, 274, 443B

Bullock, J. S., Kolatt, T. S., Sigad, Y., Sommerville, R. S., Kravtsov, A. V., Klypin, A. A., Primack, J. R., \& Dekel, A. 2001, MNRAS, 321, 559

Busha, M. T., Adams, F. C., Evrard, A. E., \& Wechsler, R. H. 2003, ApJ, 596, 713

Busha, M. T., Evrard, A. E., Adams, F. C., \& Wechsler, R. H. 2005, MNRAS, 363, L11

Cloín, P., Avila-Reese, V., \& Valenzuela, O. 2000, ApJ, 542, 622

Davis, M., Efstathiou, G., Frenk, C. S., \& White, S. D. M. 1985, ApJ, 292, 371D

Diemand, J., Moore, B., \& Stadel, J. 2005, Nature, 433, 389

Doroshkevice, A. G., \& Zel'dovich, Ia. B. 1975, Ap\&SS, 35, 55

Eke, V. R., Cole, S., \& Frenk, C. S. 1996, MNRAS, 282, 263

Eke, V. R., Navarro, J. F., \& Steinmetz, M. 2001, ApJ, 554, 114

Evrard, A. E., \& Crone, M. M. 1992, ApJ, 394, L1

Evrard, A. E. et al. , 2002 ApJ, 573, 7E

Gao, L, White, S. D. M., Jenkins, A., Frenk, C. S., \& Springel, V. 2005, MNRAS, 363, 379

Jenkins, A., Frenk, C. S., Pearce, F. R., Thomas, P. A., Colberg, J, M., White, S. D. M., Couchman, H. M. P., Peacock, J. A., Efstathiou, G., \& Nelson, A. N. 1998, ApJ, 499, 20J

Jenkins, A., Frenk, C. S., White, S. D. M., Colberg, J. M., Cole, S., Evrard, A. E., Couchman, H. M. P., \& Yoshida, N. 2001, MNRAS, 321, 372
Katz, N., Quinn, T., \$ Gelb, J. 1993, MNRAS, 265, 689

Klypin, A., Kravtsov, A., Valenzuela, O., \& Pradda, F. 1999, ApJ, 522, 82K

Knebe, A., Devriendt, J. E. G., Mahmood, A., \& Silk, J. 2002, MNRAS, 329, 813

Knebe, A., Devriendt, J. E. G., Gibson, B. K., \& Silk, J. 2003, MNRAS, 345, 1285

Lynden-Bell, D. 1967, MNRAS, 136, 101

Ma, C., \& Boylan-Kolchin, M. 2004, PhRvL, 93b, 1301

Moore, B., Quinn, T., Governato, F., Stadel, J., \& Lake, G. 1999. MNRAS, 310, 1147

Nagamine, K., \& Loeb, A. 2003, NewA, 8, 439

Navarro, J. F., Frenk, C. S., \& White S.D.M. 1997, ApJ, 490, 493 (NFW)

Peebles, P.J.E. 1980, The Large-Scale Structure of the Universe (Princeton: Princeton Univ. Press)

Perlmutter, S., et al. 1998, ApJ, 517, 565

Power, C., Navarro, J. F., Frenk, C. S., White, S. D. M., Springel, V., Stadel, J., \& Wuinn, T. 2003, MNRAS, 338, 14.

Press, W. H., \& Schechter, P. 1974, ApJ, 318, 425

Seljak, U., \& Zaldarriaga, M. 1996, ApJ, 496, 437

Sheth, R. K., \& Tormen, G. 1999, MNRAS, 308, 119

Sommer-Larsen, J., \& Dolgov, A. 2001, ApJ, 551, 608S

Spergel, D. N., et al. 2003, ApJS, 148, 175

Spergel, D. N., et al. (astro-ph/0603449 submitted ApJ)

Springel, V. 2005, MNRAS, 364, 1105

Springel, V., White, S. D. M., Guiseppe, T., \& Kauffmann, G. 2001, MNRAS, 328, 726

Thompson, L. A., Gregory, S. A. 1978, ApJ, 220, 809

Wechsler, R. H., Bullock, J. S., Primack, J. R., Kravtsov, A. V., \& Dekel, A. 2002, ApJ, 568, 52 (W02)

White, M., Croft, R. A. C. 2000, ApJ, 539, 497

White, S. D. M. Frenk, C. S., \& Davis, M. 1983, ApJ, 274, 1

Zel'dovich, Ya. 1970, A\&A, 5, 84 